ARTICLE

\title{
GPCR kinase knockout cells reveal the impact of individual GRKs on arrestin binding and GPCR regulation
}

\author{
J. Drube (10 1,4 R. S. Haider (10 1,4, E. S. F. Matthees (1) 1, M. Reichel (1) 1, J. Zeiner ${ }^{2}$, S. Fritzwanker ${ }^{3}$, C. Ziegler ${ }^{1}$,

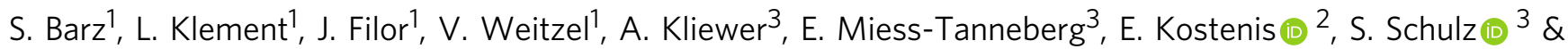 \\ C. Hoffmann (i) ${ }^{1 凶}$
}

G protein-coupled receptors (GPCRs) activate $\mathrm{G}$ proteins and undergo a complex regulation by interaction with GPCR kinases (GRKs) and the formation of receptor-arrestin complexes. However, the impact of individual GRKs on arrestin binding is not clear. We report the creation of eleven combinatorial HEK293 knockout cell clones lacking GRK2/3/5/6, including single, double, triple and the quadruple GRK knockout. Analysis of $\beta$-arrestin1/2 interactions for twelve GPCRs in our GRK knockout cells enables the differentiation of two main receptor subsets: GRK2/3-regulated and GRK2/3/5/6-regulated receptors. Furthermore, we identify GPCRs that interact with $\beta$-arrestins via the overexpression of specific GRKs even in the absence of agonists. Finally, using GRK knockout cells, PKC inhibitors and $\beta$-arrestin mutants, we present evidence for differential receptor- $\beta$-arrestin1/2 complex configurations mediated by selective engagement of kinases. We anticipate our GRK knockout platform to facilitate the elucidation of previously unappreciated details of GRK-specific GPCR regulation and $\beta$ arrestin complex formation.

\footnotetext{
${ }^{1}$ Institut für Molekulare Zellbiologie, CMB - Center for Molecular Biomedicine, Universitätsklinikum Jena, Friedrich-Schiller-Universität Jena, Hans-KnöllStraße 2, D-07745 Jena, Germany. ${ }^{2}$ Molecular, Cellular and Pharmacobiology Section, Institute for Pharmaceutical Biology, University of Bonn, Nussallee 6, 53115 Bonn, Germany. ${ }^{3}$ Institut für Pharmakologie und Toxikologie, Universitätsklinikum Jena, Friedrich-Schiller-Universität Jena, Drackendorfer Straße 1, D-07747 Jena, Germany. ${ }^{4}$ These authors contributed equally: J. Drube, R. S. Haider. ${ }^{凶}$ email: carsten.hoffmann@med.uni-jena.de
} 
G protein-coupled receptors (GPCRs) constitute the largest family of membrane receptors in human physiology comprising more than 800 identified members. GPCRs regulate multitudes of physio- and pathophysiological processes and are well-established targets for pharmacological intervention. A recent review listed 134 GPCRs and about 50 additional GPCRsignalling related proteins which are directly targeted by Food and Drug Administration-approved drugs ${ }^{1}$.

The diverse stimuli recognised by GPCRs induce conformational changes within the receptor, which activate distinct signalling pathways ${ }^{2}$. As opposed to the large number of GPCRs, the intracellular signalling molecules are less diverse. Besides G proteins, GPCR kinases (GRKs) and arrestins are the most immediate GPCR-interacting molecules ${ }^{3,4}$. The human genome contains seven genes that encode for different GRK isoforms: GRK1-7. The two visual GRKs (GRK1 and 7) are specifically expressed in the retina to facilitate the shutoff of photopigment signalling. Similarly, the expression of GRK4 predominantly occurs in specific tissues (e.g. testis or the heart) ${ }^{5}$. Of the four arrestin genes that are conserved in vertebrates (arrestin-1 or visual arrestin, arrestin- 2 or $\beta$-arrestin 1 , arrestin- 3 or $\beta$-arrestin 2 and arrestin- 4 or cone arrestin), two isoforms, namely arrestin-1 and -4 , aid in photopigment desensitisation and are restrictively expressed in rod and cone cells. Hence, the regulation of hundreds of non-visual GPCRs is hypothesised to be orchestrated by just six ubiquitously expressed proteins: four GRKs (GRK2, 3, 5 and 6) and two arrestin isoforms ( $\beta$-arrestin 1 and 2$)^{6}$.

To explain this apparent imbalance, the phosphorylation barcode hypothesis for receptor-arrestin interactions was developed ${ }^{7-9}$. Since GRK-induced GPCR phosphorylation is the basis for highaffinity $\beta$-arrestin binding, we anticipate that individual GRK isoforms shape the GPCR signalling response in a cell- and tissuespecific manner.

The relative selectivity of ligands to favour a certain pathway at the expense of others was termed functional selectivity or biased agonism $^{6,10,11}$. The recognition that either $G$ protein- or arrestinsupported pathways ${ }^{12}$ can contribute to pathophysiological conditions or drug-associated side effects ${ }^{10,13}$ triggered an intense search for biased ligands. GRKs act as essential mediators and define $\beta$ arrestin functions via ligand-specific GPCR phosphorylation or preferential coupling to certain active receptor states. Structural biology greatly contributed to our understanding of receptor conformational changes, which lead to the interaction with either $G$ proteins or arrestins. For arrestins, a multi-step GPCR binding model ${ }^{14}$ was proposed back in 1993. This mechanism involves the recognition of receptor phosphorylation ${ }^{15}$ and the engagement of the arrestin finger loop region (FLR) ${ }^{16,17}$. However, little is currently known about the impact of individual GRKs on arrestin binding.

The ubiquitous expression of GRK2, 3, 5 and 6 obscures the elucidation of the roles of individual GRKs in receptor phosphorylation. Until now, siRNA/shRNA ${ }^{18-20}$ or CRISPR/Cas9 approaches targeting only a certain subset of relevant GRKs ${ }^{21}$, and the utilisation of GRK inhibitors were the only strategies used to study their impact on living cell function. Yet, in combination with phosphosite-specific antibodies ${ }^{22,23}$ or mass spectrometry ${ }^{24}$, contributions of individual GRKs to the phosphorylation of certain receptors were elucidated to some degree ${ }^{8}$.

Nevertheless, the remaining expression of the targeted GRK(s) in knockdown approaches, or potential off-target effects of pharmacological intervention preclude the unambiguous interpretation of obtained results. Thus, a comprehensive elucidation of single GRK contributions to the arrestin-dependent regulation of GPCR signalling, internalisation and trafficking remains elusive.

In this study, we present a cellular platform to investigate the individual roles of GRK2, 3, 5 and 6 in these processes. We have created a panel of eleven combinatorial HEK293 GRK knockout clones, which enable us to analyse the GRK contributions to GPCR phosphorylation, recruitment of $\beta$-arrestin 1 and 2 , as well as receptor internalisation in unprecedented detail.

\section{Results}

GRK knockout cells: a viable cellular platform to assess individual GRK contributions. Utilising the CRISPR/Cas9 technology, we engineered HEK293 single-cell clones with knockouts (KO) of GRKs. We created single KOs of GRK2 ( $\triangle \mathrm{GRK} 2)$, GRK3 ( $\Delta$ GRK3), GRK5 ( $\Delta$ GRK5), GRK6 ( $\Delta$ GRK6), two double KOs $\Delta \mathrm{GRK} 2 / 3$ and $\Delta \mathrm{GRK} 5 / 6$ and a quadruple $\mathrm{KO}$ of GRK2, 3, 5 and 6 $(\Delta \mathrm{Q}-\mathrm{GRK})$. Additionally, we established four triple $\mathrm{KO}$ cell clones $(\Delta \mathrm{GRK} 3 / 5 / 6, \Delta \mathrm{GRK} 2 / 5 / 6, \Delta \mathrm{GRK} 2 / 3 / 6$ and $\Delta \mathrm{GRK} 2 / 3 / 5)$ with the endogenous expression of one remaining GRK. To compare experiments using these KOs, we furthermore subjected HEK293 cells to the CRISPR/Cas9 process without the addition of any gRNAs (Control).

The KOs were confirmed by Western blot analysis (Fig. 1a) and further validated by the functional studies contained in this manuscript. Morphology as revealed by phase-contrast microscopy of cultured cells and cell growth (Supplementary Fig. 1a-c) were only mildly affected in some of the clones. Expression levels of the untargeted GRKs in the obtained cell clones remained virtually unchanged compared to Control (Supplementary Fig. 1d). Notably, we did not assess the expression levels of other kinases that might influence GPCR regulation, thus we cannot exclude that the presented cell lines feature the expression of e.g. GRK1, 4 and/or 7.

In order to test the effect of our $\Delta G R K$-clones and to investigate potentially altered kinase activity of GRKs untargeted in a specific KO cell line, we revisited and analysed agonist promoted $\mu$-opioid receptor (MOP) phosphorylation. This receptor system was deliberately chosen, as GRK contributions were already extensively studied 21,25 and the abundance of available phosphosite-specific antibodies allowed for complete elucidation of [D-Ala2, N-MePhe4, Gly-ol]-enkephalin (DAMGO)-induced receptor phosphorylation (Supplementary Fig. 2a, b). We successfully identified $\mathrm{T}^{376}$ as a specific target of GRK2 and 3. In line with our previous findings ${ }^{25}, \mathrm{~T}^{370}, \mathrm{~S}^{375}$ and $\mathrm{T}^{379}$ seem to be phosphorylated by all four GRKs albeit to different extents. In $\Delta \mathrm{Q}-\mathrm{GRK}$, the phosphorylation of these sites was completely abolished, confirming their role as GRK target sites. In contrast, $\mathrm{S}^{363}$, a known PKC phosphorylation site $\mathrm{s}^{25,26}$ retained its strong phosphorylation signal in $\triangle \mathrm{Q}-\mathrm{GRK}$ cells (Supplementary Fig. 2a, b). This analysis confirms the general functionality of our clones for phosphorylation studies and underlines the unaltered activity of kinases not targeted in our $\mathrm{KO}$ approach.

Further, we investigated MOP internalisation in Control, $\Delta \mathrm{GRK} 2 / 3, \Delta \mathrm{GRK} 5 / 6$ and $\Delta \mathrm{Q}$-GRK stably expressing the receptor by confocal microscopy and surface enzyme-linked immunosorbent assay (ELISA) (Supplementary Fig. 2c, d). After stimulation, MOP was internalised in Control and $\Delta$ GRK5/6, but remained at the cell surface in $\Delta \mathrm{GRK} 2 / 3$ and $\Delta \mathrm{Q}-\mathrm{GRK}$. Our findings confirm that all four analysed GRKs are able to act on the MOP, but exclusively GRK2 and 3 are able to phosphorylate $T^{376}$ and further drive the internalisation of the receptor.

GRK2, 3, 5 and 6 are able to individually induce the formation of b2AR- $\boldsymbol{\beta}$-arrestin complexes. As the availability of tools for the analysis of site-specific receptor phosphorylation is limited across the GPCR superfamily, we utilised the universal GPCR adaptor proteins $\beta$-arrestin 1 and 2 to analyse the contributions of individual GRKs to receptor regulation. The schematic in Fig. $1 \mathrm{~b}$ 
a

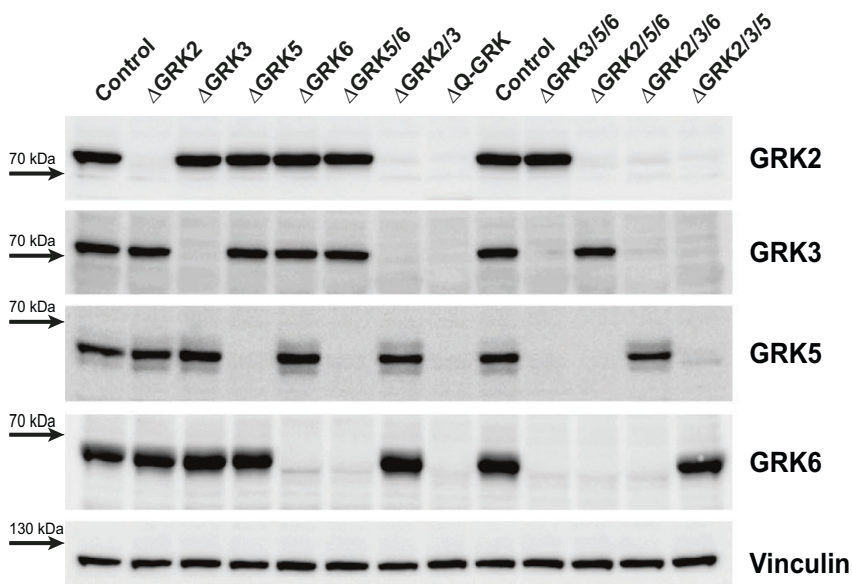

b

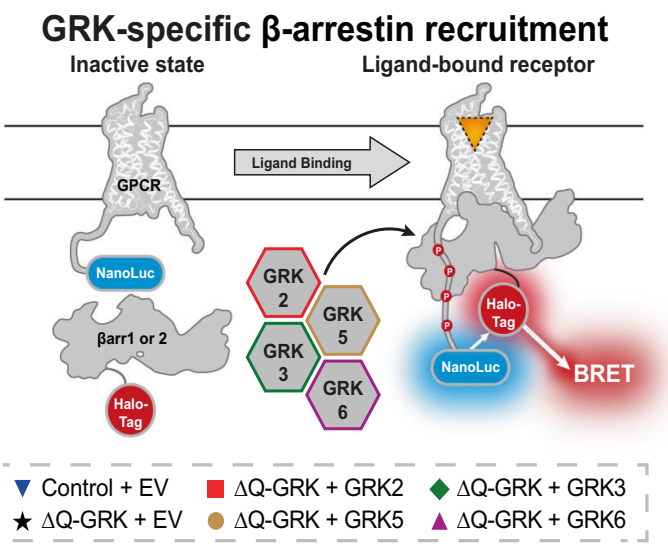

C

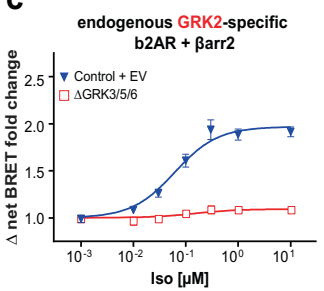

Iso $[\mu \mathrm{M}]$

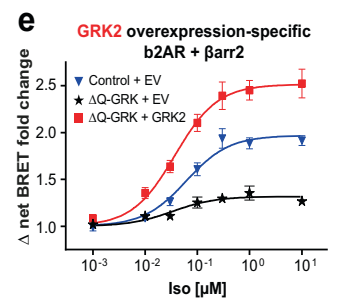

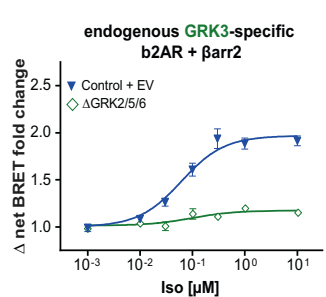

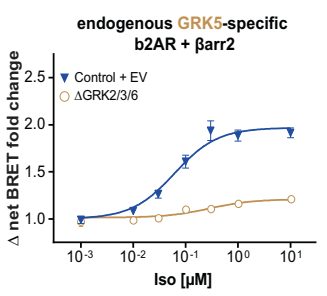

Iso [uM]
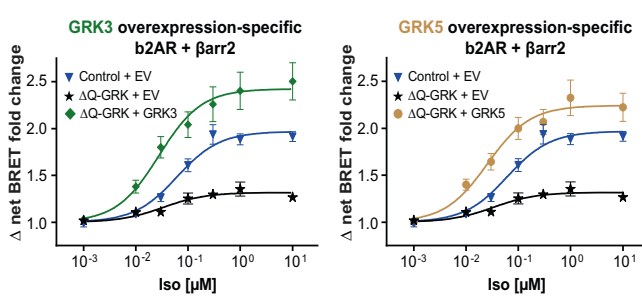
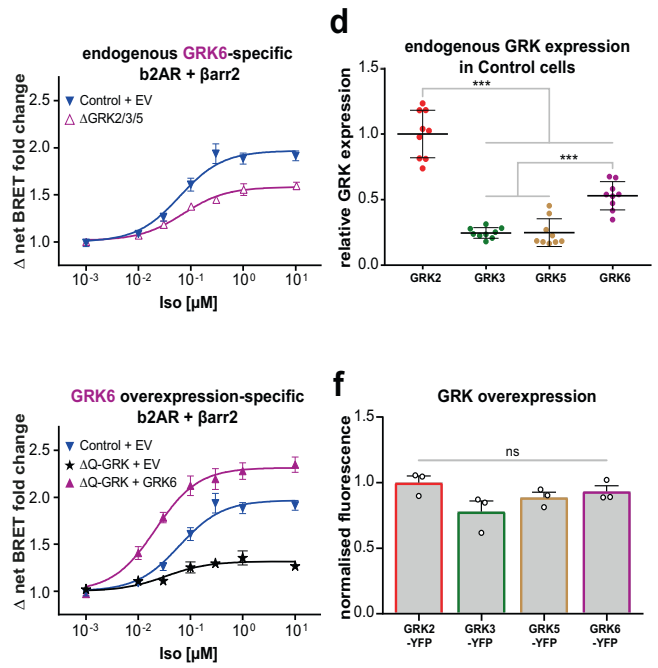

Fig. 1 GRK knockout cells enable the characterisation of GRK-specific $\boldsymbol{\beta}$-arrestin recruitment. a Single $(\Delta \mathrm{GRK} 2,3,5$ and 6$)$, double $(\Delta \mathrm{GRK} 2 / 3$ or $5 / 6)$, triple $(\Delta$ GRK3/5/6, 2/5/6, 2/3/6 and 2/3/5) and quadruple ( $\Delta$ Q-GRK) GRK knockout cells were generated using the CRISPR/Cas9 technology and established as single-cell clones. The absence of GRK2, 3, 5 or 6 was confirmed by Western blot analysis. A representative blot of $n=4$ independent experiments is shown (for quantification, see Supplementary Fig. 1d). b Schematic depiction of the performed NanoBRET $\beta$-arrestin ( $\beta$ arr) recruitment assay and colour-coding for GRK-specific conditions used throughout the paper. The Halo-Tag-ßarr fusion protein is recruited to a NanoLuciferase (NanoLuc)-tagged GPCR upon agonist activation and subsequent receptor phosphorylation. The resulting change in proximity of the Halo-Tag and the NanoLuc increases measured BRET ratios, enabling the agonist concentration-dependent analysis of $\beta$ arr recruitment. c Halo-Tag- $\beta$ arr2 recruitment to the b2AR-NanoLuc upon stimulation with isoprenaline (Iso) in cells expressing all endogenous GRKs (Control + empty vector (EV)) or only one remaining endogenous GRK (triple knockout cells $\Delta$ GRK3/5/6, $\Delta$ GRK2/5/6, $\Delta$ GRK2/3/6 and $\Delta$ GRK2/3/5). d The relative GRK protein expression in Control cells determined by Western blot of $n=3$ independent lysates as described in Reichel et al. ${ }^{27}$. Data are depicted as mean \pm SEM of $n=3$ independent blots. GRK expression levels were compared using ANOVA and two-sided Tukey's test ( $\left.{ }^{\star} p<0.05 ;{ }^{\star \star} p<0.01 ;{ }^{* \star \star} p<0.001\right)$. e $\beta$ arr 2 recruitment to the b2AR in quadruple GRK knockout cells ( $\triangle \mathrm{Q}-\mathrm{GRK}$ ), overexpressing a single GRK ( $\Delta$ Q-GRK + GRK) or expressing all endogenous GRKs (Control + EV). BRET data in (c) and (e) are presented as $\Delta$ net BRET fold change, mean of $n=3$ independent experiments \pm SEM. For better comparison, the Control and $\Delta$ Q-GRK curves are shown multiple times. $\mathbf{f}$ GRK-YFP fusion proteins were transfected in $\Delta$ Q-GRK and YFP fluorescence was measured to confirm similar expression levels of all transfected GRKs. YFP fluorescence was compared using ANOVA and two-sided Tukey's test (ns not significant). Corresponding experiments, confirming the catalytic activity of GRK-YFP fusion constructs are shown in Supplementary Fig. 3a. Measured fluorescence is depicted as a mean of $n=3$ independent experiments + SEM as normalised fluorescence. All exact $p$ values, test statistics, effect sizes, confidence intervals and degrees of freedom are provided in the Source Data files.

depicts the established bioluminescence resonance energy transfer (BRET)-based in cellulo $\beta$-arrestin recruitment assay, allowing us to reveal functional, GRK-specific GPCR phosphorylation. In this BRET assay, a NanoLuciferase (NanoLuc) is fused to the receptor C-terminus and serves as the energy donor in order to observe the association of $\beta$-arrestin constructs tagged with a Halo-Tag and labelled with the Halo 618 ligand.

First, we studied the GRK-specific interactions between the $\beta 2$ adrenergic receptor (b2AR) and $\beta$-arrestin2 utilising the endogenous expression of GRKs in various $\triangle \mathrm{GRK}$ cells. At endogenous expression levels of all four GRKs (Control), $\beta$ arrestin 2 showed clear isoprenaline (Iso)-induced recruitment to the b2AR (Fig. 1c). In comparison, $\beta$-arrestin recruitment was substantially reduced when recorded in triple GRK KO cell lines, only featuring the endogenous expression of one individual GRK (Fig. 1c, $\Delta$ GRK3/5/6, $\Delta$ GRK2/5/6, $\Delta$ GRK2/3/6 and $\Delta$ GRK2/3/5). While endogenous expression of either GRK2, 3 or 5 induced only minimal BRET changes, these were, nevertheless, sufficient to detect a ligand-dependent increase in $\beta$-arrestin recruitment. To evaluate whether recorded BRET data actually describes a 
molecular GPCR- $\beta$-arrestin interaction, all recruitment data in this study have been subjected to statistical analysis (Supplementary Table 1). Hence, only those data sets that show a significant increase for the condition stimulated with the highest ligand concentration compared to vehicle addition, are further interpreted as functional $\beta$-arrestin recruitment. Additionally, concentration-response curves were only fitted for these conditions. The highest amount of $\beta$-arrestin 2 recruitment to the b2AR was found in $\Delta \mathrm{GRK} 2 / 3 / 5$ cells, specifically induced by the endogenous expression of GRK6.

These data have to be evaluated in the context of endogenous GRK expression levels in Control cells. Hence, we conducted Western blot analysis to assess the relative endogenous expression levels of GRK2, 3, 5 and 6 as elaborated in Reichel et al. ${ }^{27}$ and found that the cytosolic GRK2 and the membrane-associated GRK6 are the most abundant (Fig. 1d). Interestingly, we identified GRK6 as the main mediator of Iso-promoted $\beta$ arrestin2 recruitment to the b2AR (Fig. 1c), when measured under the endogenous expression of GRKs. This suggests that specific GRK isoforms exhibit different affinities for coupling to the same GPCR and require differential expression levels to facilitate functional receptor regulation.

Since these findings specifically reflected on the affinities and endogenous expression levels of GRKs, we analysed the molecular capability of each individual GRK to induce b2AR- $\beta$-arrestin2 complex formation via re-introduction into $\Delta \mathrm{Q}-\mathrm{GRK}$ (Fig. 1e). The relative expression of transfected GRKs was assessed fluorometrically (Fig. 1f). Via the introduction of a C-terminal YFP fusion into the identical vector backbone and subsequent equimolar transfection of GRK-YFP constructs, we confirmed similar expression levels of the transfected kinases. To allow for this comparison, the GRK-YFP fusion proteins were characterised with at least the same capability to mediate GPCR- $\beta$ arrestin interactions as their untagged counterparts, used in all other experiments (Supplementary Fig. 3a). Additionally, the relative degree of overexpression was quantified via Western blot analysis (Supplementary Fig. 3b, c). Using this controlled overexpression of individual GRKs in $\triangle \mathrm{Q}$-GRK, all four kinases showed a similar effect on b2AR regulation: each individual GRK isoform enhanced the $\mathrm{b} 2 \mathrm{AR}-\beta$-arrestin recruitment to higher levels than induced by the combined endogenous expression of GRKs in Control cells (Fig. 1e). Interestingly, we still encountered measurable $\beta$-arrestin2 recruitment in the absence of GRKs $(\Delta \mathrm{Q}$ GRK $+E V$ ). This could be explained by the inherent affinity of $\beta$ arrestin 2 towards ligand-activated, yet unphosphorylated GPCRs.

These findings clarify that all four tested GRKs are able to individually mediate high-affinity $\beta$-arrestin 2 binding to the b2AR and that their relative tissue expression ultimately defines their specific contributions to this process.

Since all GRKs have been shown to induce similar levels of $\beta$ arrestin recruitment, we investigated whether isoform-specific phosphorylation of the b2AR might still have a pronounced effect on the conformational changes that occur during arrestin activation. To address this, we overexpressed each individual GRK isoform alongside the untagged b2AR and an intramolecular $\beta$-arrestin2-FlAsH5-NanoLuc BRET biosensor. We found comparable $\beta$-arrestin2 conformational changes for all GRKs (Fig. 2a). Note, that an equivalent FRET sensor was published previously ${ }^{28}$ and that more details on the intramolecular BRET sensor will be published elsewhere.

We further utilised the b2AR, as a model receptor regulated by all four tested GRK isoforms, to test the effect of endogenous ligands and pharmacological inhibition on GRK-specific $\beta$ arrestin-coupling processes. The application of the endogenous ligands epinephrine and norepinephrine resulted in overall lower GRK-specific $\beta$-arrestin 2 recruitment to the b2AR as compared to
Iso (Supplementary Fig. 4a, b compare with Fig. 1e). Although the relative efficacies of individual GRKs to mediate epinephrine- and norepinephrine-induced $\beta$-arrestin2 binding was unchanged in comparison to Iso ( $\Delta \mathrm{Q}-\mathrm{GRK}+\mathrm{GRK}>\mathrm{Control}+\mathrm{EV}>\Delta \mathrm{Q}$ GRK $+E V$ ), we observed a left shift of the measured concentration-response curves specifically for GRK6 (Fig. 2b-d). This significant increase in potency to elicit $\beta$-arrestin2 recruitment was observed for both endogenous ligands, although it was shown that epinephrine acts as a full agonist, whereas norepinephrine only partially activates the $\mathrm{b} 2 \mathrm{AR}^{29}$. This might have implications for the tissue-specific regulation of $\mathrm{b} 2 \mathrm{AR}$, as lower ligand concentrations might be sufficient to desensitise the receptor in tissues with relatively higher GRK6 expression.

Since we were able to measure GRK-specific $\beta$-arrestin recruitment, we hypothesise that this assay is also suitable to characterise the specificity of GRK inhibitors in a cellular system. Indeed, we were able to record the concentration-dependent inhibition of $\beta$-arrestin 2 recruitment to the receptor by cmpd101 (a known GRK2 family inhibitor) only in cells expressing GRK2 or 3 (Fig. 2e). This demonstrates cmpd101 selectivity by the lack of inhibition in cells overexpressing GRK5 or 6. When performing the analogous experiment using pindolol as a potent antagonist of the b2AR, we recorded an inhibition of $\beta$-arrestin2 recruitment regardless of GRK (over-) expression (Fig. 2f). Thus, we present a cell-based GRK-inhibitor screening platform utilising $\Delta \mathrm{Q}-\mathrm{GRK}$.

$\Delta$ Q-GRK cells reveal GRK-specificity of $\beta$-arrestin1 and 2 recruitment to different GPCRs. To investigate the GRKspecificity of GPCR regulation, we compared the individual molecular capabilities of GRK2, 3, 5 and 6 to facilitate $\beta$-arrestin recruitment across 12 different GPCRs: angiotensin II type 1 receptor (AT1R), b2AR, b2AR with an exchanged C-terminus of the vasopressin 2 receptor (b2V2), complement 5a receptor 1 (C5aR1), muscarinic acetylcholine receptors (M1R, M2R, M3R, M4R and M5R), MOP, parathyroid hormone 1 receptor (PTH1R) and vasopressin 2 receptor (V2R). This receptor panel was deliberately selected to feature receptors with divergent lengths of intracellular loop 3 (IL3, ranging from 3 to 213 amino acids) and C-termini (ranging from 8 to 105 amino acids), predominant coupling to different $G$ proteins (Supplementary Table 2), as well as GPCRs that have been shown to be additionally regulated by second messenger kinases.

As representative examples of our findings, the GRK-selective $\beta$-arrestin1 and 2 recruitment to the M5R and PTH1R are depicted in Fig. 3a, b and Fig. 3c, d, respectively (data for all receptors are shown in Supplementary Figs. 5 and 6). Both receptors were able to induce robust, agonist-dependent $\beta$ arrestin1 and 2 recruitment in Control cells, which was significantly reduced in $\triangle \mathrm{Q}-\mathrm{GRK}$. In the case of the M5R, $\beta$ arrestin1 recruitment was completely abolished in $\Delta \mathrm{Q}-\mathrm{GRK}$. Still, a major difference in GRK-selectivity of the two receptors was found using this approach: the individual overexpression of GRK2, 3, 5 and 6 significantly increased $\beta$-arrestin recruitment to the PTH1R in $\triangle \mathrm{Q}-\mathrm{GRK}$, whereas GRK5 and 6 were unable to facilitate M5R- $\beta$-arrestin complex formation. These findings were additionally confirmed in triple GRK KO cell lines and the $\Delta \mathrm{GRK} 2 / 3$ and $\Delta \mathrm{GRK} 5 / 6$ family KOs (Supplementary Fig. 7a, b). Interestingly, the endogenous expression of GRK2 and 3 in $\Delta \mathrm{GRK} 3 / 5 / 6$ and $\Delta \mathrm{GRK} 2 / 5 / 6$ was sufficient to increase the measured $\beta$-arrestin2 recruitment in comparison to $\Delta \mathrm{Q}-\mathrm{GRK}$ for both receptors. This finding essentially confirms the functionality of these two triple GRK KO cell lines and suggests that the M5R and PTH1R require lower amounts of GRK2 or 3 in order to be efficiently regulated in comparison to the b2AR 
a
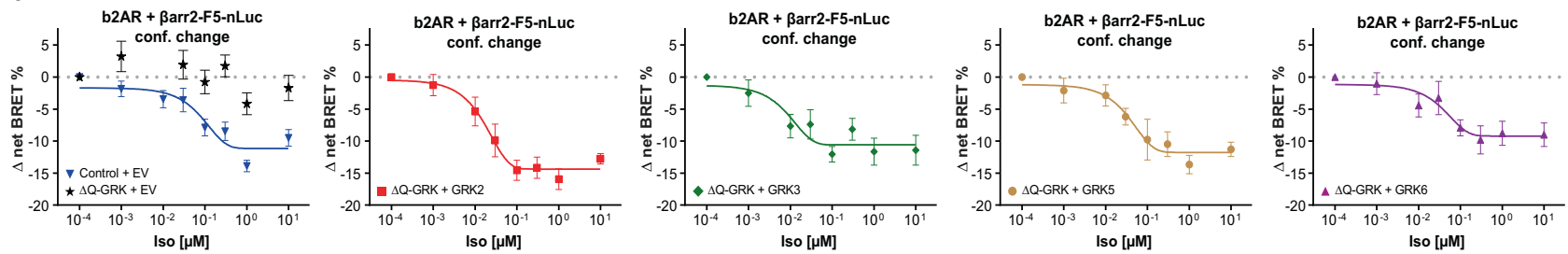

b

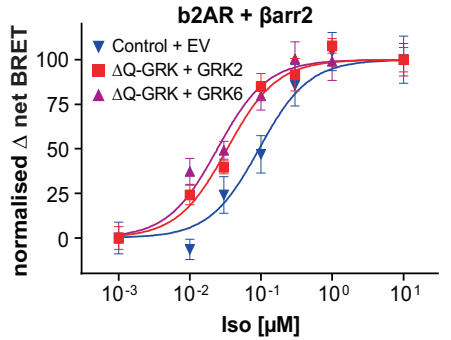

\begin{tabular}{lccll}
\hline \multicolumn{1}{c}{ Isoprenaline } & $\begin{array}{c}\mathrm{EC}_{50} \\
(\mu \mathrm{M})\end{array}$ & SEM & p-value \\
\hline Control + EV & 0.095 & \pm 0.027 & & \\
$\Delta \mathrm{Q}-\mathrm{GRK}+\mathrm{GRK} 2$ & 0.037 & \pm 0.012 & $0.013 \quad$ & $*$ \\
$\Delta \mathrm{Q}-\mathrm{GRK}+\mathrm{GRK} 6$ & 0.026 & \pm 0.009 & 0.006 & $* *$ \\
\hline
\end{tabular}

c

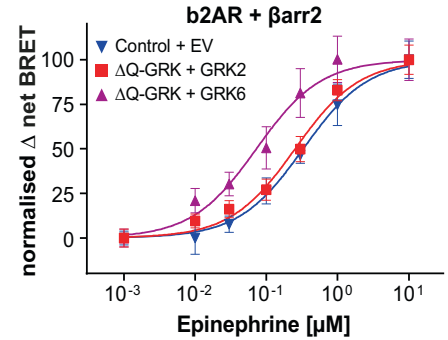

\begin{tabular}{lcccl}
\hline \multicolumn{1}{c}{ Epinephrine } & $\begin{array}{c}\mathrm{EC}_{50} \\
(\boldsymbol{\mu M})\end{array}$ & SEM & \multicolumn{1}{c}{ p-value } \\
\hline Control + EV & 0.349 & \pm 0.134 & & \\
$\Delta \mathrm{Q}-\mathrm{GRK}+$ GRK2 & 0.323 & \pm 0.130 & $0.945 \quad \mathrm{~ns}$ \\
$\Delta \mathrm{Q}-\mathrm{GRK}+$ GRK6 & 0.090 & \pm 0.035 & 0.049 & * \\
\hline
\end{tabular}

d

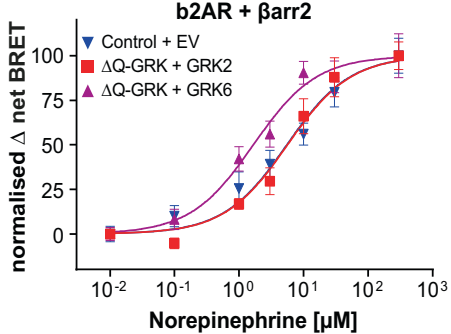

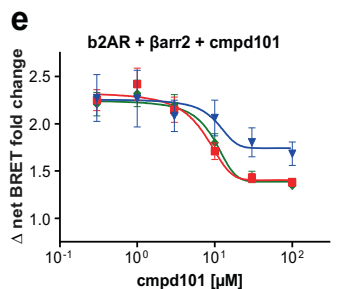

cmpd101 $[\mu \mathrm{M}]$
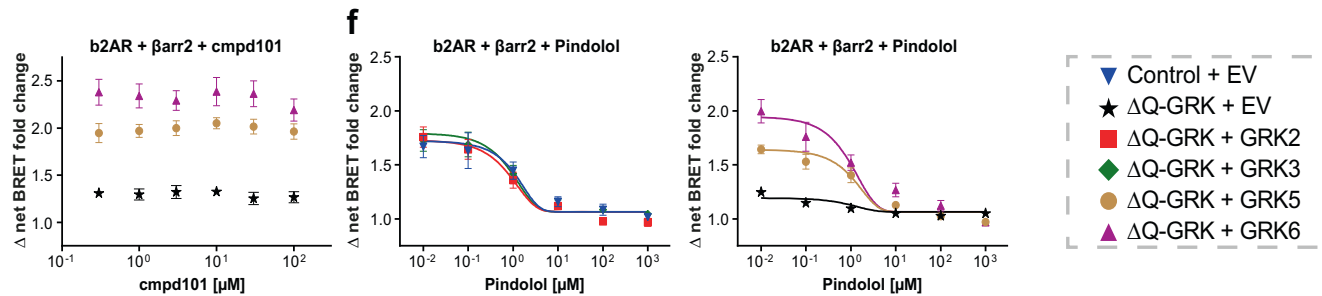

Fig. 2 GRK knockout cells are a viable cellular platform to assess various features of b2AR- $\beta$-arrestin complex formation. a Analysis of $\beta$-arrestin 2 ( $\beta$ arr2) conformational changes. $\Delta \mathrm{Q}-\mathrm{GRK}$ or Control cells were transfected with an untagged b2AR expression construct and the $\beta$ arr2-F5-NanoLuc

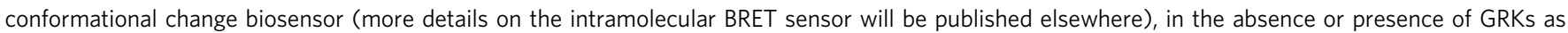
noted and stimulated with isoprenaline (Iso). Conformational change data are shown as $\Delta$ net BRET change in per cent, mean of $n=3$ independent

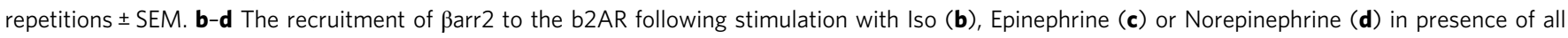
endogenous GRKs or individually overexpressed GRK2 or GRK6 in $\Delta$ Q-GRK as indicated. Data are depicted as a mean of $n=3$ independent experiments \pm SEM and normalised to individual maxima. The tables below depict the $\mathrm{EC}_{50} \pm \mathrm{SEM}$ of the corresponding concentration-response curves. The $\mathrm{EC}_{50}$ of the indicated conditions were compared to the $\mathrm{EC}_{50}$ in Control using ANOVA and two-sided Dunnett's test ( ${ }^{\star} p<0.05$; ${ }^{\star \star} p<0.01$; ns not significant). e, $\mathbf{f}$ Utilisation of the $\beta$ arr recruitment assay for specificity determination of the GRK inhibitor $\mathrm{cmpd101}$ in living cells. $\Delta \mathrm{Q}-\mathrm{GRK}$ or Control cells

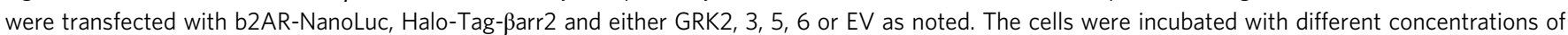
cmpd101 (e) or the b2AR antagonist pindolol (f) for 10 min prior to stimulation with $1 \mu \mathrm{M}$ Iso. The recruitment-induced BRET changes were measured and calculated as $\Delta$ net BRET change in per cent, represented as the mean of $n=4$ independent experiments \pm SEM. All exact $p$ values, test statistics, effect sizes, confidence intervals and degrees of freedom are provided in the Source Data files.

(Fig. 1c). As indicated by the experiments shown in Fig. 3b, $\Delta$ GRK cell lines only featuring the expression of GRK5 and/or 6 did not increase the $\beta$-arrestin2 recruitment to the M5R as in comparison to $\triangle \mathrm{Q}-\mathrm{GRK}$.

Further, we employed confocal live-cell microscopy to assess the dependency of PTH1R and M5R internalisation on endogenous GRK levels in Control, $\Delta \mathrm{GRK} 2 / 3$ and $\Delta \mathrm{GRK} 5 / 6$ as well as in $\Delta \mathrm{Q}$-GRK. Under basal conditions, $\beta$-arrestin 2 is located in the cytosol, M5R and PTH1R in the cell membrane and Rab5 (early endosome marker) in endosomes (Fig. 3e, $\mathrm{f}$ basal). As expected, the M5R was not able to induce $\beta$-arrestin 2 translocation in the absence of GRK2 and $3(\Delta \mathrm{GRK} 2 / 3$ and $\Delta$ Q-GRK) (Fig. 3e).

The quantification of co-localisation between the M5R and $\beta$ arrestin2 (Fig. 3g) confirms our findings of Fig. $3 \mathrm{~b}$ and Supplementary Fig. 7a. Analysis of M5R co-localisation with Rab5 (as a surrogate measurement for receptor internalisation and initial trafficking) reveals that this interaction translates to functional receptor internalisation only in the presence of GRK2 and 3 (Fig. 3e, g). For the PTH1R, we were able to detect ligandinduced co-localisation with $\beta$-arrestin 2 or Rab5 in all conditions expressing GRKs (Fig. 3f, h). Interestingly, the agonist-stimulated PTH1R was still able to induce a slight membrane translocation of $\beta$-arrestin 2 in $\Delta \mathrm{Q}-\mathrm{GRK}$, confirming the GRK-independent affinity of $\beta$-arrestin2 toward the ligand-activated receptor (Fig. 3d). The results obtained for the PTH1R using endogenous GRK expression were verified in a reciprocal experiment overexpressing single GRKs in $\triangle \mathrm{Q}$-GRK for $\beta$-arrestin1 and 2 (Supplementary Fig. 7c-f).

These apparent differences in GRK-specific $\beta$-arrestin recruitment, as exemplified by the M5R and the PTH1R, were encountered multiple times during our analysis across twelve different GPCRs (Supplementary Fig. 5). Via statistical multiple comparisons of BRET fold changes at saturating ligand concentrations for each of the tested 
a

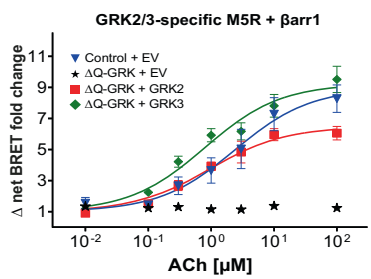

b

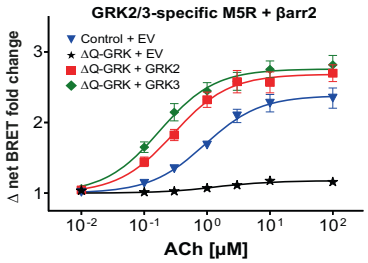

e

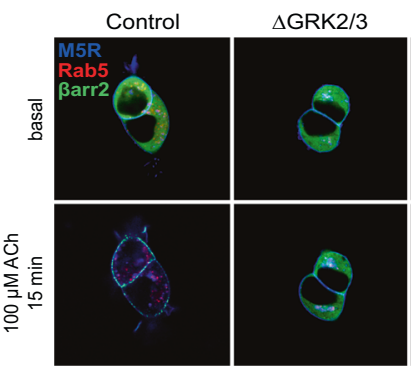

g
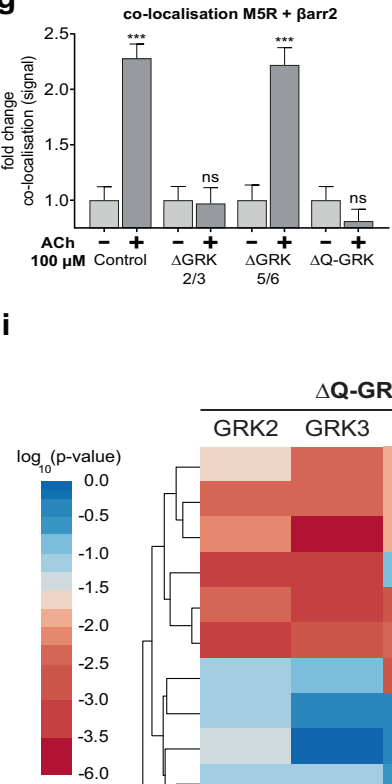
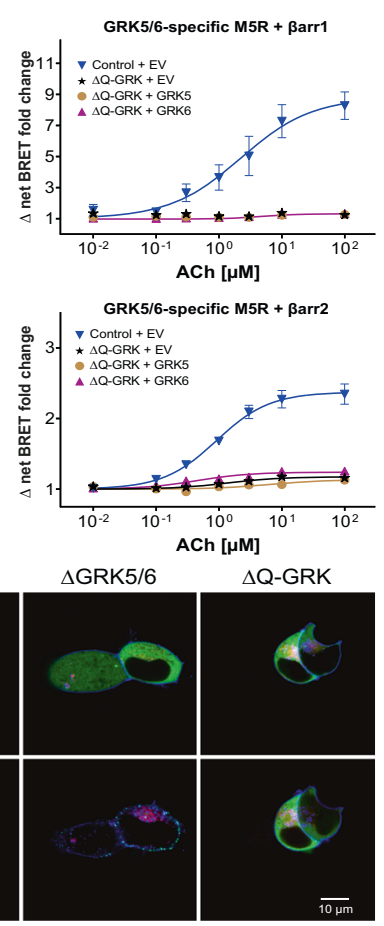

co-localisation M5R + Rab5

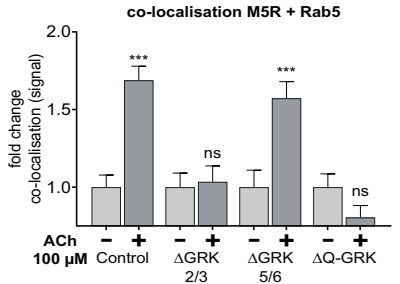

c

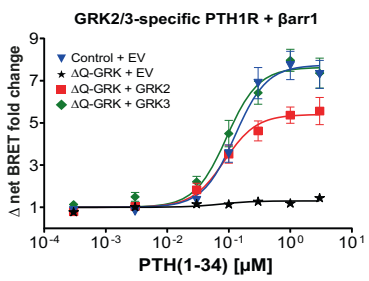

d

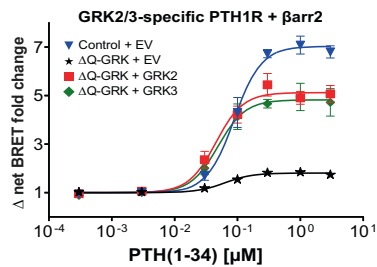

Control

f

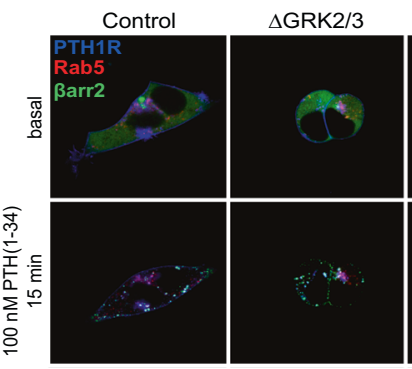

h
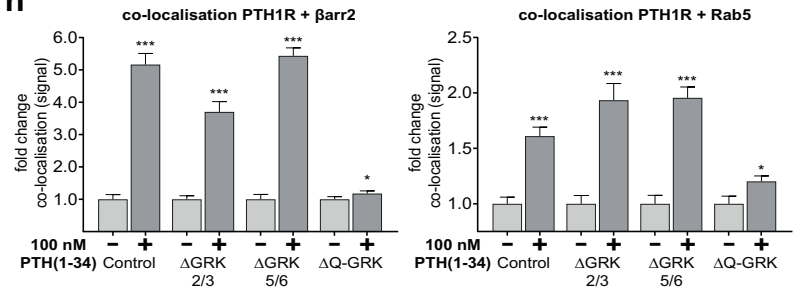

j
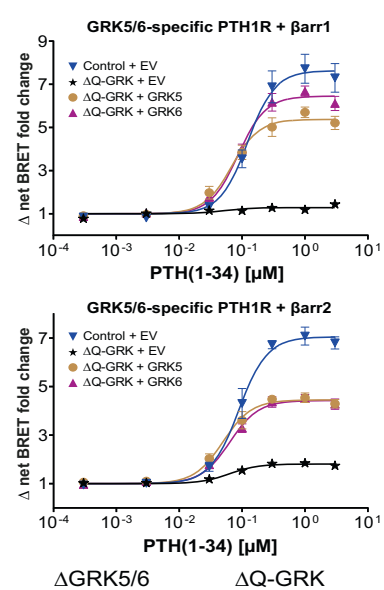

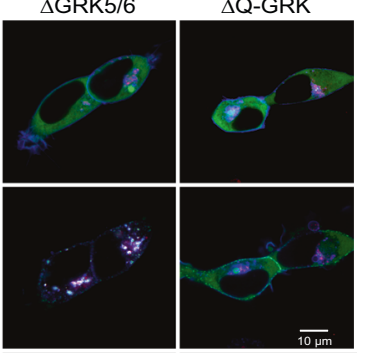

co-localisation PTH1R + Rab5

\section{Overview of GRK-mediated $\beta$-arrestin interactions}
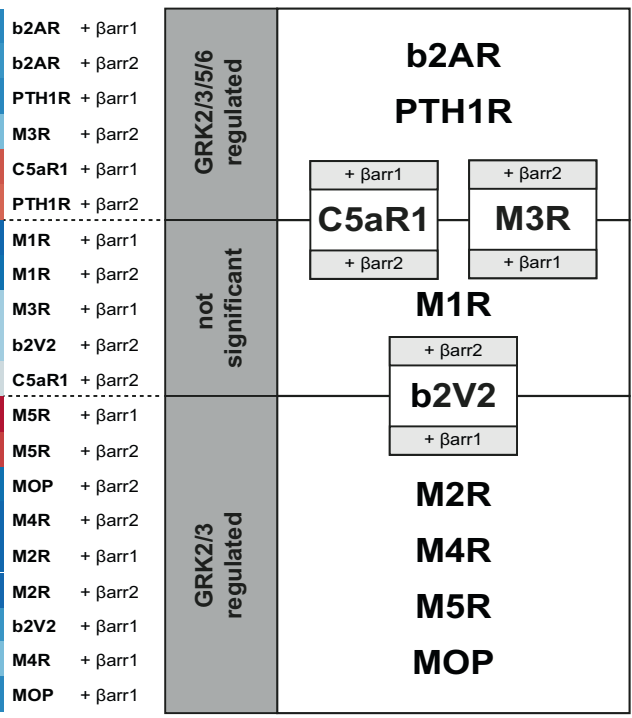

conditions (Control $+\mathrm{EV}, \Delta \mathrm{Q}-\mathrm{GRK}+\mathrm{EV}, \Delta \mathrm{Q}-\mathrm{GRK}+\mathrm{GRK} 2, \Delta \mathrm{Q}-$ GRK + GRK3, $\Delta \mathrm{Q}-\mathrm{GRK}+\mathrm{GRK} 5$ and $\Delta \mathrm{Q}-\mathrm{GRK}+\mathrm{GRK} 6)$, we were able to cluster the respective GPCR- $\beta$-arrestin pairs into groups, depending on the found GRK-selectivity (Fig. 3i, j; Supplementary Table 3). Here, we identified several subsets of GPCRs: receptors for which $\beta$-arrestin interaction is mediated by overexpression of (i) any
GRK (b2AR, PTH1R, C5aR1 $+\beta$-arrestin1 and M3R $+\beta$-arrestin2) or (ii) GRK2 or 3 only (M2R, M4R, M5R, MOP and b2V2+ $\beta$-arrestin1). Within our tested GPCRs, we did not observe $\beta$-arrestin interaction mediated exclusively by GRK5 or 6 . A third group is comprised of receptor- $\beta$-arrestin pairs, which did not consistently show significant differences between the tested conditions and hence 
Fig. $3 \Delta$ Q-GRK cells reveal GRK-specificity of $\beta$-arrestin1 and 2 recruitment to different GPCRs and allow assessment of GRK-dependent GPCR internalisation and $\boldsymbol{\beta}$-arrestin2 translocation. $\mathbf{a}-\mathbf{d}$ GRK-specific $\beta$-arrestin ( $\beta$ arr)1 (a, $\mathbf{c}$ ) or $\beta$ arr2 (b, d) recruitment to the M5R upon acetylcholine (ACh) stimulation (a, b) or the PTH1R upon parathyroid hormone 1-34 (PTH(1-34)) stimulation (c, d). Shown are concentration-response curves depicted as $\Delta$ net BRET fold change, mean of $n=3$ independent experiments \pm SEM. The panels display the recruitment in presence of either GRK2 or 3 or GRK5 or 6 . For better comparison, the Control and $\Delta$ Q-GRK curves are shown multiple times. e, $\mathbf{f}$ Control, $\Delta$ GRK2/3, $\Delta$ GRK5/6 and $\Delta$ Q-GRK cells were transfected with either M5R-CFP or PTH1R-CFP (blue), the early endosome marker Rab5-mCherry (red) and $\beta$ arr2-YFP (green) expression constructs. The cells were grown on coverslips and subjected to confocal live-cell microscopy. Shown are representative images, taken before and after 15 min of stimulation with either $100 \mu \mathrm{M}$ ACh or $100 \mathrm{nM}$ PTH(1-34), respectively. The normalised co-localisation of M5R (g) or PTH1R (h) with $\beta$ arr2 or Rab5 was quantified using Squassh and SquasshAnalyst (number of images per respective condition; Control: M5R (39), PTH1R (38); $\Delta$ GRK2/3: M5R (35), PTH1R (32); $\Delta$ GRK5/6: M5R (36), PTH1R (33); $\triangle$ Q-GRK: M5R (38), PTH1R (55)). Data are presented as mean fold change in co-localisation signal + SEM. Statistical analysis was performed using a two-way mixed model ANOVA followed by a two-sided paired $t$-test ( ${ }^{\star} p<0.05$; ${ }^{\star \star} p<0.01 ;{ }^{\star \star \star} p<0.001$; ns not significant). i Clustering heatmap representing the statistical multiple comparisons of $\beta$ arr recruitment data for ten different GPCRs. Conditions with overexpressed GRKs were tested against $\Delta \mathrm{Q}-\mathrm{GRK}+$ empty vector (EV) or Control $+\mathrm{EV}$, as indicated. Additionally, $\Delta \mathrm{Q}-\mathrm{GRK}+\mathrm{EV}$ as compared to Control $+\mathrm{EV}$. BRET fold changes at saturating ligand concentrations of at least $n=3$ independent experiments were compared using ANOVA and two-sided Bonferroni's test (Supplementary Table 3, data derived from Supplementary Fig. 5). Transformed unadjusted $p$ values are plotted. GPCR- $\beta$ arr pairs are clustered according to Canberra distance. $\mathbf{j}$ Overview of clustering from $\mathbf{i}$ in GPCR- $\beta$ arr pairs regulated by any tested GRK (GRK2/3/5/6 regulated), by GRK2 or 3 only (GRK2/3 regulated) and a third group, which is comprised of GPCR- $\beta$ arr pairs that do not consistently show significant differences between the tested conditions. All exact $p$ values, test statistics, effect sizes, confidence intervals and degrees of freedom are provided in the Source Data files.

could not be definitively assigned to one of the first two groups $(\mathrm{M} 1 \mathrm{R}, \mathrm{C} 5 \mathrm{aR} 1+\beta$-arrestin2, $\mathrm{M} 3 \mathrm{R}+\beta$-arrestin1 and $\mathrm{b} 2 \mathrm{~V} 2+\beta$ arrestin2). In case of C5aR1 $+\beta$-arrestin2 (Supplementary Fig. $5 \mathrm{~d}$ ) this behaviour is explained by exceptionally high $\beta$-arrestin 2 recruitment in the absence of GRKs.

Interestingly, two distinct GPCRs, namely the AT1R and V2R, evaded the statistical grouping process. Both receptors exhibited apparently diminished, agonist-dependent $\beta$-arrestin recruitment in the presence of certain overexpressed GRKs as compared to their effects in $\Delta$ Q-GRK (Supplementary Fig. $5 \mathrm{~g}, \mathrm{~m}$ ). This finding was highly unexpected, therefore we further focussed on the elucidation of these GRK-dependent processes.

GRK2, 3, 5 or 6 individually enable ligand-independent $\beta$ arrestin1 and 2 interactions with the V2R. Besides previous intensive studies ${ }^{30,31}$, the V2R and AT1R stood out unique in our in-depth, GRK subtype-specific $\beta$-arrestin recruitment assay (Fig. 4, Supplementary Fig. $5 \mathrm{~m}$ ). In the presence of all four endogenously expressed GRKs (Control), agonist stimulation induced clear recruitment of $\beta$-arrestin1 and 2 to the V2R (Fig. 4a, b; Supplementary Fig. 8a, b). In their absence ( $\Delta$ Q-GRK) the recruitment of $\beta$-arrestins was reduced, as expected. Surprisingly, individual overexpression of GRK2, 3, 5 or 6 did not further increase the concentration-dependent, dynamic BRET change of the interaction. When comparing the respective BRET ratios measured before and after stimulation at ligand saturation (Fig. 4a, b; Supplementary Fig. 8a, b), we found that already the basal BRET ratios were remarkably increased in presence of overexpressed GRKs. This was unlike any other receptor investigated in Fig. 3i (Supplementary Figs. 5 and 6).

The concentration-response curves shown to this point reflect the fold change between the measured baseline and stimulated BRET ratios. The elevated baselines explain the unexpectedly low dynamic BRET changes, even though the absolute values of stimulated BRET ratios show a clear increase in the presence of overexpressed GRKs. Thus, we conclude that already the basal molecular interaction between the V2R and $\beta$-arrestins is increased under those conditions.

Two major interaction interfaces between GPCRs and $\beta$ arrestins have been proposed. Namely, the interaction mediated by phosphorylated intracellular domains of the receptor (e.g. C-terminus and IL3) only ("hanging" complex) ${ }^{17,32}$, as well as the additional insertion of the arrestin FLR into the intracellular cavity of the GPCR ("core" complex) ${ }^{16}$. Since we expected the unstimulated V2R to be in an inactive conformation, we hypothesised that the interaction between $\beta$-arrestins and the intracellular cavity of the GPCR is prevented and therefore occurs in a "hanging" conformation. If this hypothesis was correct, deletion of the $\beta$-arrestin FLR should not impair the measured association with V2R. Thus, we analysed $\beta$-arrestin recruitment with biosensors lacking the FLR ( $\beta$-arrestin1/2-dFLR) (Fig. $4 c$, d; Supplementary Fig. 8c, d).

Indeed, for $\beta$-arrestin1-dFLR the baseline BRET measurements remained elevated, while agonist stimulation was not able to further increase the interaction between $\beta$-arrestin1-dFLR and the V2R (Fig. 4c, d). Thus, we propose ligand-independent precoupling of $\beta$-arrestin1 to the V2R in a "hanging" complex in presence of overexpressed GRKs. The remaining liganddependent increase in $\beta$-arrestin1 recruitment might be explained by ligand-activation of the pre-coupled "hanging" complex and subsequent engagement of the FLR to form a tight "core" complex (Fig. $4 \mathrm{~g}$ ). In contrast, $\beta$-arrestin 2 pre-coupling was found to depend on the FLR (Supplementary Fig. 8c, d). These findings show that $\beta$-arrestin 1 interacts with the V2R using an association with the $\mathrm{C}$-terminus whereas $\beta$-arrestin2 seems to require both interactions, including the association of the FLR with the transmembrane helix bundle.

However, the kinase-dependent pre-coupling of $\beta$-arrestins to the receptor was not observed for the chimeric b2V2 (Supplementary Fig. 6). Hence, we conclude that the C-terminus is not solely responsible for the mediation of this effect.

Via confocal microscopy, we observed that the individual overexpression of GRK2 and 6 significantly increased the ligandindependent co-localisation between the V2R and Rab5 or $\beta$ arrestin1 in comparison to $\triangle \mathrm{Q}-\mathrm{GRK}$ (Fig. 4e, f; Supplementary Fig. $8 \mathrm{e}-\mathrm{h})$. This confirms that ligand-independent V2R- $\beta$-arrestin interactions, as facilitated by overexpressed GRKs, lead to functional receptor internalisation in line with the observations of Snyder et al. ${ }^{33}$. Furthermore, experiments conducted with GRK2 and 6 kinase-dead (KD; K220R, K215R, respectively) mutants (Supplementary Fig. 9) support that pre-coupling of $\beta$-arrestin1 in presence of overexpressed GRKs is, in fact, dependent on their kinase activity.

Distinct AT1R- $\beta$-arrestin complex configurations are mediated by GRK2/3, GRK5/6 or PKC. Multiple groups already investigated different AT1R- $\beta$-arrestin interactions ${ }^{34,35}$. However, we found that the GRK-specificity of $\beta$-arrestin complex configurations was even more intricate for the AT1R in comparison to the V2R. Therefore, we arranged the data obtained for the AT1R in a kinasespecific manner in Fig. 5a-f. We observed the most prominent 


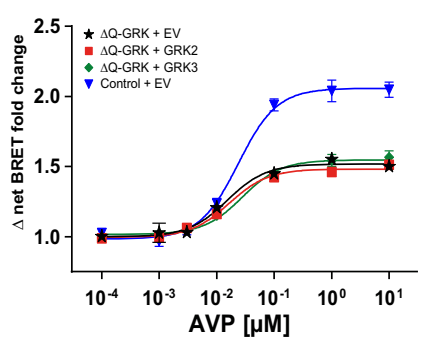

GRK2/3-specific V2R + ßarr1

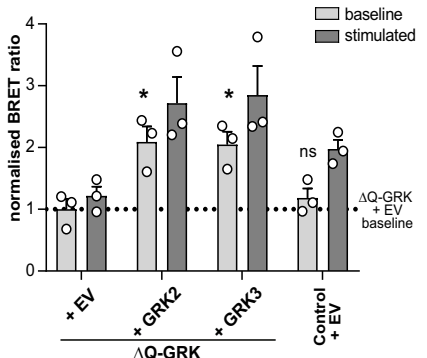

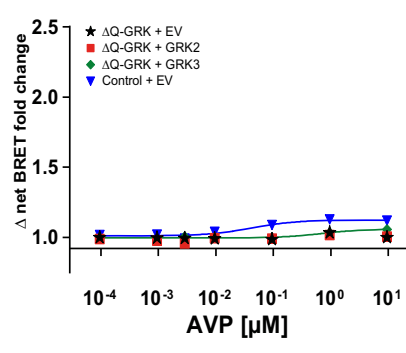

GRK2/3-specific V2R + $\beta$ arr1-dFLR

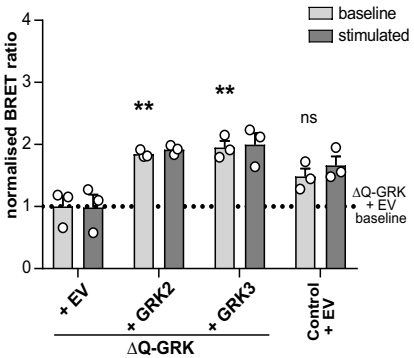

b
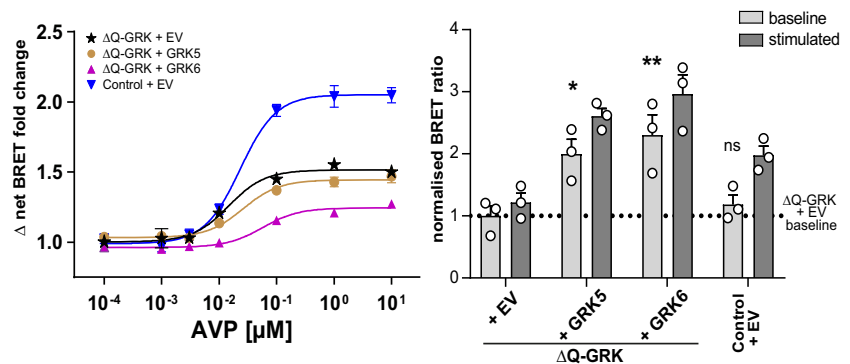

d
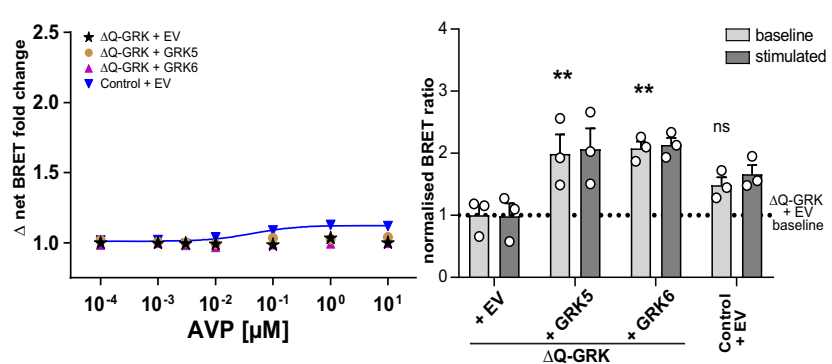

e

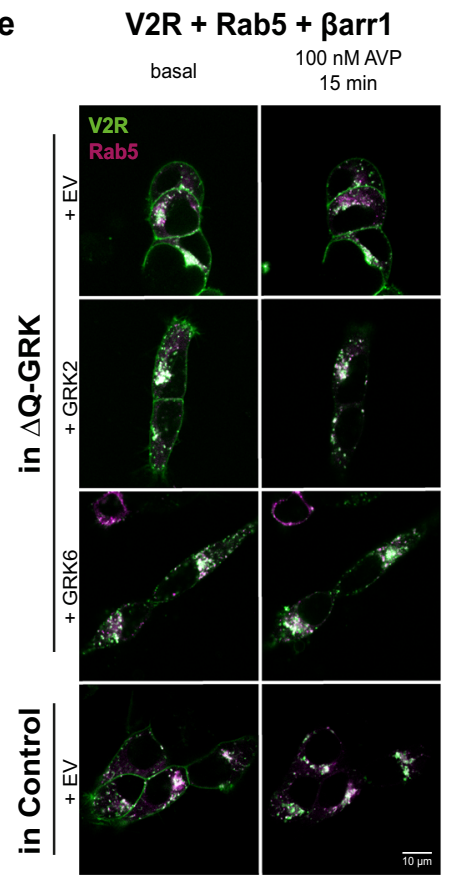

f Microscopy analysis: V2R + Rab5 + ßarr1
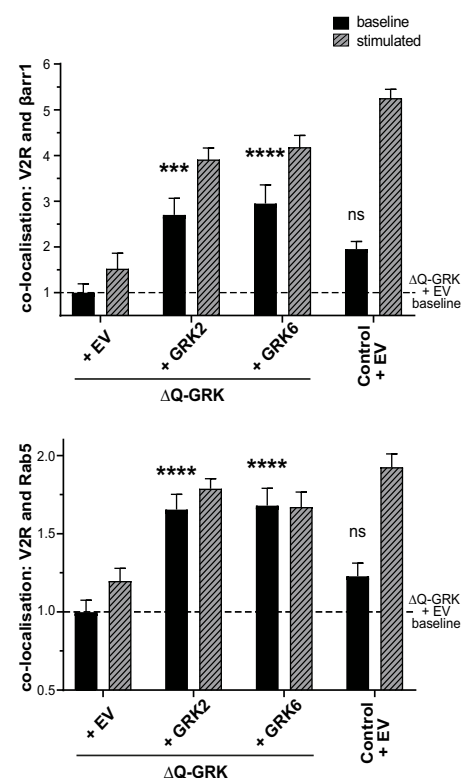

g GRK-dependent ßarr1 coupling to V2R

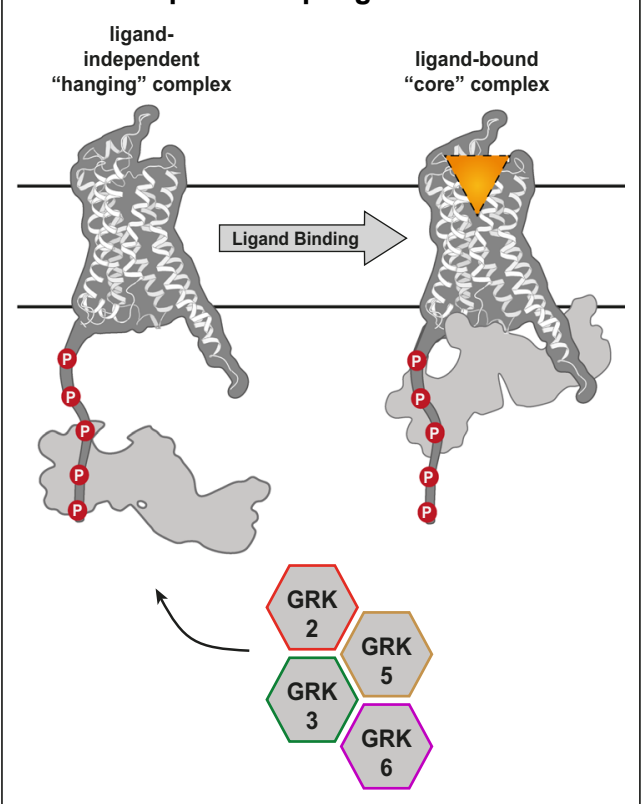

difference between angiotensin II (AngII)-induced $\beta$-arrestin1 and 2 recruitment to the $\mathrm{AT} 1 \mathrm{R}$, in $\triangle \mathrm{Q}-\mathrm{GRK}$, as this condition features pronounced higher recruitment of $\beta$-arrestin2 (Supplementary Fig. 5g and Supplementary Fig. 6). Since the AT1R is a known target of heterologous desensitisation ${ }^{30}$, we anticipated that PKC could be responsible for mediating this difference ${ }^{35}$ at this Gq-coupled receptor. Hence, we conducted the experiment in the presence of Gö6983, a pan PKC inhibitor. This reduced the recruitment of both $\beta$-arrestins in Control cells and abolished GRK-independent $\beta$ arrestin1 recruitment (Supplementary Figs. 10a, b and 11a, b). In contrast, PKC inhibition had a negligible effect on the dynamic $\beta$ arrestin recruitment in the presence of overexpressed GRKs. Moreover, the overexpression of GRK5 and 6 showed lower dynamic recruitment of $\beta$-arrestin2 in comparison to $\triangle \mathrm{Q}$-GRK, regardless of PKC activity (Fig. 5e, f; Supplementary Fig. 10a, b). Taken together, we conclude that dynamic GRK-mediated $\beta$-arrestin2 recruitment to the AT1R is unaffected by PKC activity, whereas for $\beta$-arrestin1 this is promoted by both GRKs and PKC.

Again, analysis of the measured BRET ratios reveals significantly increased basal molecular interaction in the presence of overexpressed GRK5 and 6 (Fig. 5e, f; Supplementary Fig. 6). This finding identifies the AT1R as yet another receptor that interacts with $\beta$-arrestins in a ligand-independent fashion, similar to the V2R. Interestingly, in this specific case, GRK5 and 6 seem to be able to account for the observed interactions. Notably, our statistical analysis shows a significant increase for the baseline of GRK3-mediated $\beta$-arrestin recruitment as well. However, since the greater part of the recruitment seems to be ligand-dependent in this condition, we did not conclude efficient AT1R- $\beta$-arrestin pre-coupling mediated by GRK3. 
Fig. 4 GRK2, 3, 5 or $\mathbf{6}$ can individually mediate a ligand-independent interaction of the V2R and $\boldsymbol{\beta}$-arrestin1. a-d $\Delta \mathrm{Q}$-GRK or Control cells were transfected with V2R-Halo-Tag and one of the following $\beta$-arrestin1 ( $\beta$ arr1)-NanoLuc fusion constructs: wild type (a, b) or $\beta$ arr1 lacking the finger loop region ( $\mathrm{dFLR} ; \mathbf{c}, \mathbf{d})$. Additionally, either $\mathrm{GRK} 2,3,5,6$ or the empty vector (EV) were transfected as indicated. The dynamic BRET changes are shown as ligand concentration-response curves normalised to baseline values and vehicle control. All data points are calculated as $\Delta$ net BRET fold change, mean of $n=3$ independent experiments \pm SEM. The same dataset is presented as bar graphs, displaying the mean BRET values + SEM before (baseline) and after stimulation with $10 \mu \mathrm{M}\left[\mathrm{Arg}^{8}\right.$ ]-vasopressin (AVP; stimulated), normalised to the basal BRET ratio derived from the $\Delta \mathrm{Q}-\mathrm{GRK}+\mathrm{EV}$ condition (dashed line). To test whether the baseline BRET ratios were significantly elevated compared to the respective $\triangle \mathrm{Q}-\mathrm{GRK}+\mathrm{EV}$ baseline, an ANOVA and one-sided Dunnett's test was performed $\left({ }^{\star} p<0.05 ;{ }^{\star \star} p<0.01\right.$; ns not significant; $\mathbf{a}, \mathbf{b} \Delta \mathrm{Q}-\mathrm{GRK}+\mathrm{GRK} 2 p=0.0113, \Delta \mathrm{Q}-\mathrm{GRK}+\mathrm{GRK} 3 p=0.0142, \Delta \mathrm{Q}-\mathrm{GRK}+\mathrm{GRK} 5$ $p=0.0188, \Delta \mathrm{Q}-\mathrm{GRK}+\mathrm{GRK} 6 p=0.0036$, Control $+\mathrm{EV} p=0.6186 ; \mathbf{c}, \mathbf{d}: \Delta \mathrm{Q}-\mathrm{GRK}+\mathrm{GRK} 2 p=0.0073, \Delta \mathrm{Q}-\mathrm{GRK}+\mathrm{GRK} 3 p=0.0033, \Delta \mathrm{Q}-\mathrm{GRK}+\mathrm{GRK} 5$ $p=0.0024, \Delta \mathrm{Q}-\mathrm{GRK}+\mathrm{GRK6} p=0.0013$, Control $+\mathrm{EV} p=0.1054)$. e, $\mathbf{f} \Delta \mathrm{Q}-\mathrm{GRK}$ or Control cells were transfected with V2R-CFP (green), Rab5-mCherry (magenta), $\beta$ arr1-YFP (not shown) and either EV, GRK2 or GRK6 as indicated. Images were taken before (basal) and after $15 \mathrm{~min}$ of $100 \mathrm{nM}$ AVP stimulation. Representative images are shown in (e) and Supplementary Fig. 8e-h. The co-localisation of V2R and $\beta$ arr1 or Rab5 was quantified using Squassh and SquasshAnalyst (number of images per respective condition; $\Delta \mathrm{Q}-\mathrm{GRK}+\mathrm{EV}$ (35), $\Delta \mathrm{Q}-\mathrm{GRK}+\mathrm{GRK2}$ (35), $\Delta \mathrm{Q}-\mathrm{GRK}+\mathrm{GRK6}$ (33),

Control + EV (30)). f Data are presented as mean fold change in co-localisation signal + SEM normalised to unstimulated (baseline) $\Delta \mathrm{Q}-\mathrm{GRK}+\mathrm{EV}$ condition. Co-localisation prior to stimulation was compared using ANOVA and two-sided Dunnett's test $\left({ }^{\star} p<0.05 ;{ }^{\star \star} p<0.01 ;{ }^{\star \star \star} p<0.001\right.$;

${ }^{\star \star \star \star} p<0.0001$; ns not significant). All exact $p$ values, test statistics, effect sizes, confidence intervals and degrees of freedom are provided in the Source Data files. $\mathbf{g}$ Schematic depiction of $\beta$ arr1 interactions with the V2R in the absence and presence of ligand, facilitated by high expression levels of GRKs.

After the discovery of these fundamental kinase-specific effects, we investigated whether this AT1R- $\beta$-arrestin complexes occur in a "hanging" or "core" configuration (Fig. 5; Supplementary Table 4). As stated above, PKC inhibition reduced AngII-induced $\beta$-arrestin2 recruitment in $\Delta \mathrm{Q}-\mathrm{GRK}$, but the recruitment is still detectable. However, deletion of the FLR abolished AngIIinduced $\beta$-arrestin2 recruitment in $\triangle \mathrm{Q}$-GRK independently of PKC activity (Fig. 5b). Thus, we conclude that the FLR is essential for the PKC-mediated AT1R- $\beta$-arrestin 2 complex, as well as a GRK- and PKC-independent complex. This suggests that PKC activity alone cannot mediate a "hanging" complex configuration.

In the presence of overexpressed GRK2 or 3, dynamic, AngIIinduced $\beta$-arrestin 2 recruitment is neither altered by $\mathrm{PKC}$ inhibition nor deletion of the FLR (Fig. $5 c$, d). Interestingly, upon PKC inhibition the measured absolute BRET ratios are decreased to the levels recorded for the AngII-induced recruitment of the dFLR construct. This led us to the assumption that PKC inhibition and the deletion of the FLR mediate the same biological effect. Since PKC activity alone was shown to only mediate a "core" complex configuration (Fig. 5b) and the FLR is dispensable for GRK2/3mediated, AngII-induced $\beta$-arrestin 2 binding, we propose that GRK2 and 3 predominantly facilitate the formation of a "hanging" complex. Despite this, with our experimental setup, we cannot exclude the formation of a "core" complex between the two proteins under physiological conditions with unaltered PKC activity.

The pre-coupling effect mediated by GRK5 and 6 (Fig. 5e, f) is also observed for the $\beta$-arrestin2-dFLR mutant, suggesting the formation of a ligand-independent "hanging" complex with $\beta$ arrestin2. Again, the remaining ligand-dependent increase in $\beta$ arrestin 2 recruitment measured for GRK5 overexpression could reflect ligand-activation of the pre-coupled "hanging" complex and subsequent formation of a tight "core" complex (Fig. 5g), similar to the mechanism proposed for the V2R (Fig. 4g). Notably, GRK6-mediated pre-coupling was reduced, indicating that the FLR plays a role in this process.

The utilisation of GRK KD mutants revealed that the precoupling of $\beta$-arrestin 2 is mediated by GRK 5 or 6 kinase activity (Supplementary Fig. 10c, e, g). Particularly, the phosphorylation of inactive AT1R in the presence of overexpressed GRK5 has been reported before ${ }^{36}$. Interestingly this $\beta$-arrestin 2 pre-coupling effect was not observed in $\Delta \mathrm{GRK} 2 / 3 / 6$ or $\Delta \mathrm{GRK} 2 / 3 / 5$ (Supplementary Fig. 10d, f), indicating that it is dependent on individual GRK expression levels. This could explain how the same receptor might be differentially regulated in specific tissues, cellular compartments ${ }^{9}$ or under pathophysiological conditions featuring dysregulated GRK expression levels ${ }^{37}$.
In general, the GRK-dependent interaction of both $\beta$-arrestin isoforms and the AT1R is similar (Supplementary Fig. 11c-f). In the case of $\beta$-arrestin1, GRK5 and 6 overexpression also led to an enhanced basal interaction with AT1R (Supplementary Fig. 11g, h). Additionally, we conclude that both $\beta$-arrestins can use PKC phosphorylation to further stabilise a "core" complex with the AT1R. Interestingly, we observed recruitment of $\beta$-arrestin 2 in absence of GRK and PKC phosphorylation, whereas $\beta$-arrestin1 does not seem to be able to interact with the unphosphorylated receptor. Thus, we can exclude the formation of a GRK- and PKC-independent "core" complex for $\beta$-arrestin1 (Fig. 5g, Supplementary Fig. 11).

Ligand-independent AT1R regulation by GRK6 leads to receptor internalisation and impaired signalling responses. To test if these different kinase effects have a direct impact on receptor functionality, we employed confocal microscopy and dynamic mass redistribution $^{38}$ (DMR) measurements. In $\triangle \mathrm{Q}$-GRK the AT1R did not show pronounced internalisation, while GRK2 overexpression strongly supports AngII-dependent receptor internalisation. In contrast, the AT1R was already found in intracellular compartments when overexpressing GRK6, independently of ligand application (Fig. 6a, b; Supplementary Fig. 12).

As expected, AngII evoked robust primary receptor signalling in GRK-deficient cells when assessed by DMR (Fig. 6c, d). Interestingly, co-transfection of $\Delta \mathrm{Q}$-GRK with $\beta$-arrestin2 did not suffice to diminish cellular AT1R signalling despite significant $\beta$ arrestin2 recruitment under comparable experimental conditions (Fig. 6a compare with 5b). Even though PKC-specific phosphorylation can stabilise AT1R- $\beta$-arrestin 2 interactions (Fig. 5b), we conclude that GRK phosphorylation is strictly required for $\beta$ arrestin2-mediated receptor desensitisation and internalisation (Fig. 6b). The cellular signalling responses were significantly dampened under GRK2 overexpression, confirming efficient receptor desensitisation in this condition. In this system GRK2 functions as a canonical sensor for receptor activation, governing location and activity of GPCRs via the mediation of $\beta$-arrestin functions.

The overexpression of GRK6 almost eliminated the measured cellular signalling response (Fig. $6 c$, d). This, in combination with the observed pattern of AT1R subcellular localisation, suggests that GRK6-mediated AT1R phosphorylation precludes a majority of receptor molecules from the membrane and thus from being exposed to the ligand. This is especially significant, as it demonstrates that the upregulation of GRKs could have two distinctly different consequences. Depending on the combination of involved kinases and receptors, it could either lead to a canonical 
a AT1R + Barr2 in Control + EV

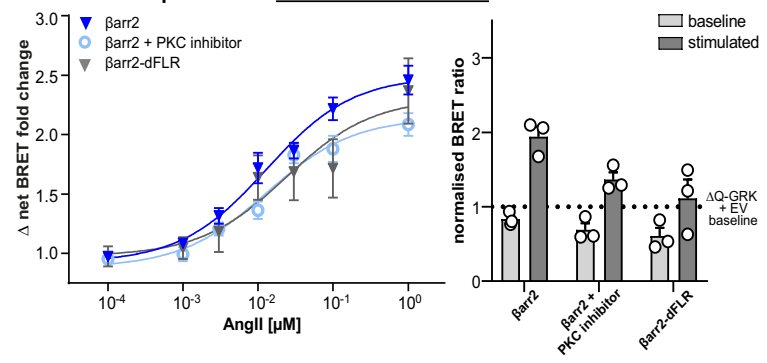

C $\mathrm{AT} 1 \mathrm{R}+$ + $\operatorname{arr} 2$ in $\triangle \mathrm{Q}-\mathrm{GRK}+\mathrm{GRK2}$
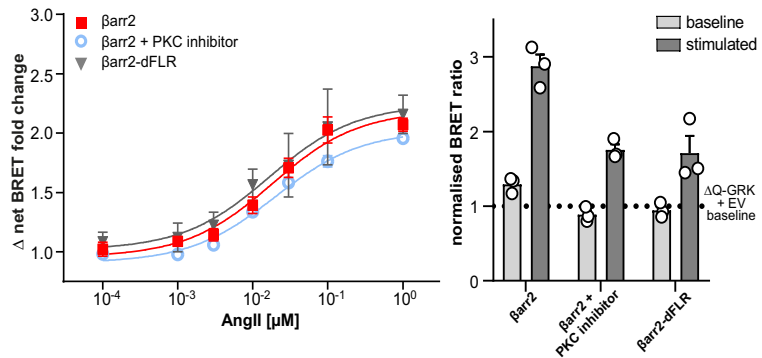

e $\mathrm{AT} 1 \mathrm{R}+$ + arr2 in $\triangle \mathbf{Q}-\mathbf{G R K}+\mathbf{G R K 5}$
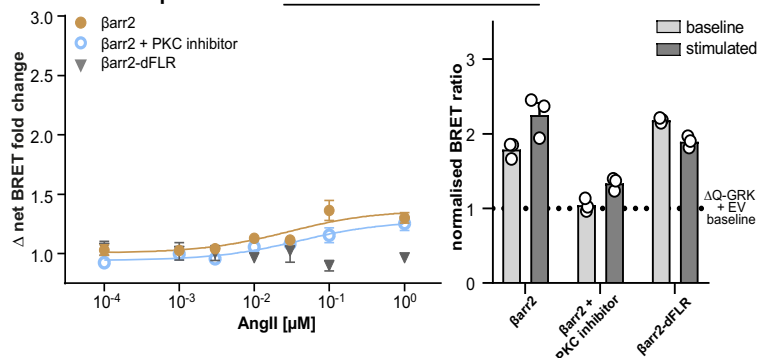

f $A T 1 R+\beta a r r 2$ in $\triangle Q$-GRK + GRK6
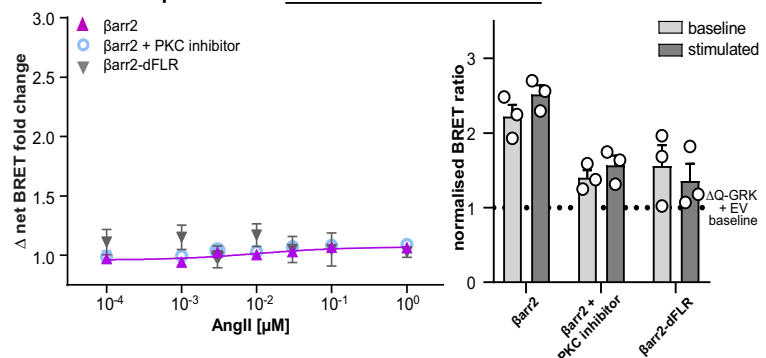

b $\quad$ AT1R + Barr2 in $\underline{\Delta Q-G R K+E V}$

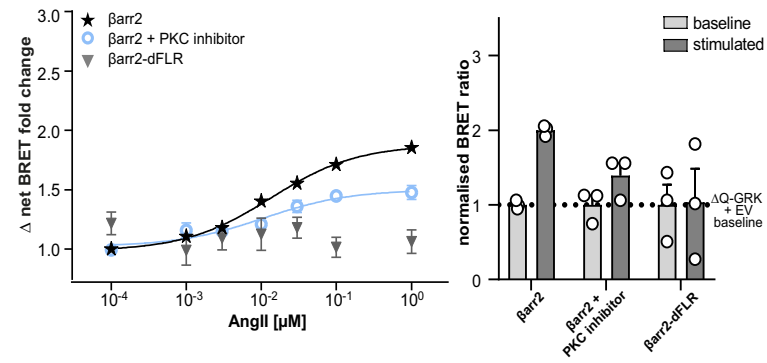

d

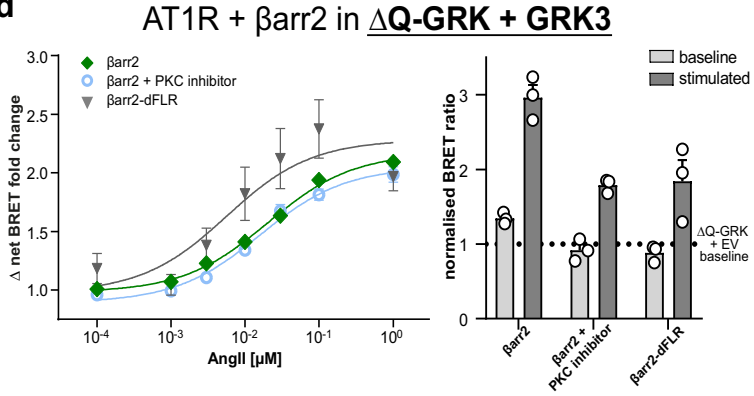

g Kinase-specific complex configurations between AT1R and Barr

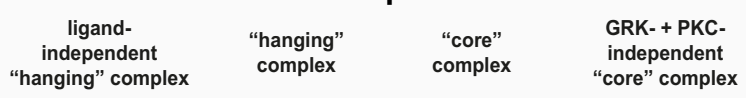

Fig. 5 Distinct AT1R- $\boldsymbol{\beta}$-arrestin2 complex configurations are mediated by GRK2/3, GRK5/6 or PKC. a-f To identify the contribution of the individual GRKs to the complex formation of $\beta$-arrestin2 ( $\beta$ arr2) with AT1R, Control or $\Delta$ Q-GRK cells were transfected with AT1R-NanoLuc, Halo-Tag- $\beta$ arr2 and either GRK2, 3, 5, 6 or the empty vector (EV) as indicated, in absence or presence of PKC inhibitor Gö6983 (500 nM). Additionally, the GRK-specific $\beta$ arr2 recruitment to the AT1R was measured utilising a Halo-Tag- $\beta$ arr2 construct lacking the finger loop region (dFLR). Angiotensin II (AngII)-induced dynamic BRET changes are shown as concentration-response curves. All data points are calculated as $\Delta$ net BRET fold change normalised to baseline values and vehicle control, represented as the mean of $n=3$ independent experiments \pm SEM. The data are also presented in respective bar graphs, displaying the mean BRET values + SEM before (baseline) and after stimulation with $1 \mu \mathrm{M}$ Angll (stimulated), normalised to the basal BRET ratio derived from the corresponding $\Delta \mathrm{Q}-\mathrm{GRK}+\mathrm{EV}$ condition in (b). The results of the statistical analysis of displayed data are listed in Supplementary Table 4. All test statistics, effect sizes, confidence intervals and degrees of freedom are provided in the Source Data files. $\mathbf{g}$ Schematic summary of the kinase-specific complex configurations between AT1R and $\beta$ arr1 or 2 either in the absence of GRKs, mediated by GRK2/3 or GRK5/6 overexpression as observed in (a-f; Supplementary Fig. 11).

increase in efficiency to induce arrestin-mediated receptor regulation or an almost complete loss of GPCR responsiveness.

\section{Discussion}

The establishment of various GRK KO cell lines enabled us to identify biological patterns of GRK-specific $\beta$-arrestin-mediated
GPCR regulation. Our comprehensive analysis revealed clustering of GPCRs into different groups, i. a. GRK2/3-regulated and GRK2/3/5/6-regulated receptors. While the V2R and AT1R are both regulated by all tested GRKs, they exhibited substantial $\beta$ arrestin pre-coupling upon GRK overexpression and therefore constitute a subgroup of GRK2/3/5/6-regulated receptors. Another conclusion that can be drawn from the presented GRK- 
a

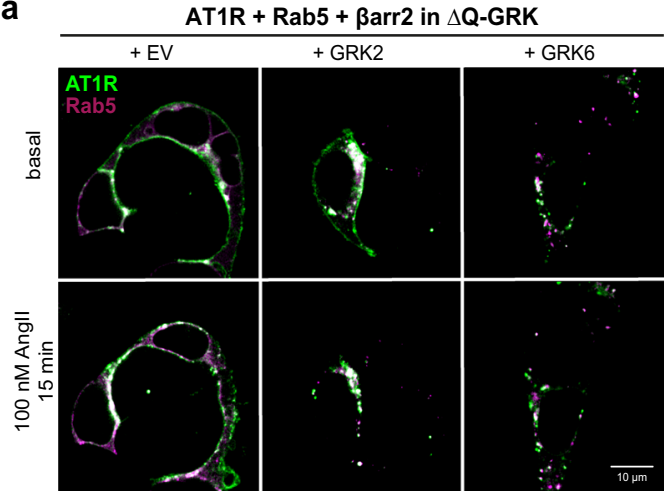

b

Microscopy analysis:

AT1R + Rab5 + ßarr2
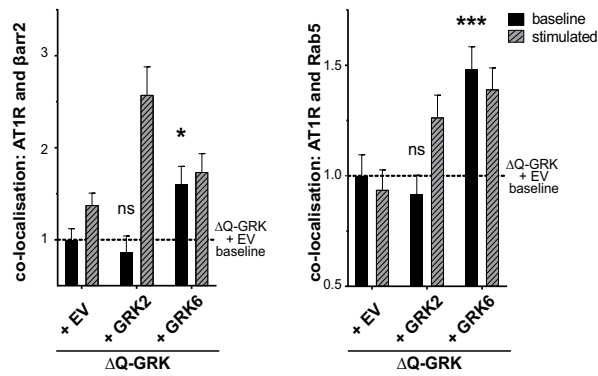

C

AT1R

AT1R + Barr2
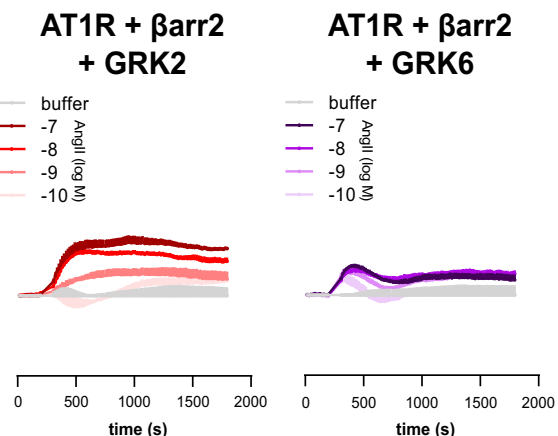

d $\quad$ AT1R in $\triangle \mathbf{Q}$-GRK

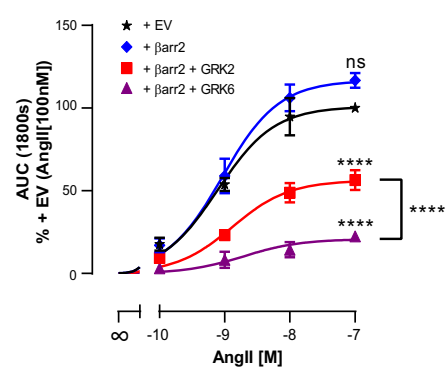

Fig. 6 Pre-coupling of $\beta$-arrestin2 mediated by overexpression of GRK6 results in desensitisation and constitutive internalisation of the AT1R. a, b $\Delta$ Q-GRK cells were transfected with hsp-AT1R-CFP (green), Rab5-mCherry (magenta), ßarr2-YFP (not shown) and either EV, GRK2 or GRK6, as noted. Images were taken before (basal) and after $15 \mathrm{~min}$ of $100 \mathrm{nM}$ Angll stimulation. Representative images are shown in (a) and Supplementary Fig. 12. The co-localisation of AT1R and Barr2 or Rab5 was quantified using Squassh and SquasshAnalyst (number of images per respective condition; $\Delta \mathrm{Q}-$ GRK + EV (31), $\Delta$ Q-GRK + GRK2 (31), $\Delta$ Q-GRK + GRK6 (33)) (b). Data are presented as mean fold change in co-localisation signal + SEM normalised to unstimulated $\Delta$ Q-GRK + EV. Co-localisation prior to stimulation was compared using ANOVA and two-sided Dunnett's test ( ${ }^{\star} p<0.05$; ${ }^{\star \star} p<0.01$; ${ }^{* \star *} p<0.001$; ns not significant; basal AT1R co-localisation with $\beta$ arr2: $\Delta \mathrm{Q}-\mathrm{GRK}+\mathrm{GRK} 2 p=0.812, \Delta \mathrm{Q}-\mathrm{GRK}+\mathrm{GRK} 6 p=0.027$; basal AT1R co-localisation with Rab5: $\Delta \mathrm{Q}-\mathrm{GRK}+\mathrm{GRK} 2 p=0.7649, \Delta \mathrm{Q}-\mathrm{GRK}+\mathrm{GRK} 6 p=0.0008)$. c $\Delta \mathrm{Q}-\mathrm{GRK}$ cells transiently transfected with hsp-AT1R-CFP, with or without cotransfection of either $\beta$ arr2 alone or in combination with GRK2 or GRK6, were stimulated with Angll and real-time dynamic mass redistribution (DMR) responses were recorded as a measure of AT1R activity. DMR recordings are shown as mean + SEM of three technical replicates from a single experiment, representative of $n=3$ independent experiments. d Concentration-effect curves derived from $n=3$ independent experiments (representatively shown in (c) $) \pm$ SEM are plotted from the area under the curve (AUC) within 0 and $1800 \mathrm{~s}$. Data are normalised to the picometre wavelength shifts evoked with $100 \mathrm{nM}$ Angll in cells expressing hsp-AT1R-CFP alone. Statistical significance was calculated using a two-way ANOVA and two-sided Tukey's test $\left({ }^{\star} p<0.05 ;{ }^{* \star} p<0.01 ;{ }^{* \star \star} p<0.001 ;{ }^{\star \star \star \star} p<0.0001\right.$; ns not significant). All exact $p$ values, test statistics, effect sizes, confidence intervals and degrees of freedom are provided in the Source Data files.

specific $\beta$-arrestin recruitment screen (Supplementary Fig. 5) is the increased ability of $\beta$-arrestin2 to form GRK-independent complexes with GPCRs, as compared to $\beta$-arrestin1. Multiple experiments in this study suggest that $\beta$-arrestin 2 exhibits higher recruitment in $\Delta \mathrm{Q}$-GRK than $\beta$-arrestin1, regardless of which GPCR was tested. This behaviour of $\beta$-arrestin2 was already hypothesised in the publication of Zhan et al. ${ }^{39}$. The authors provided the crystal structure of $\beta$-arrestin2 and showed that the protein displays higher flexibility than other arrestin isoforms. Because of this, $\beta$-arrestin2 is more likely to "probe" different inactive and active conformations, even in the absence of phosphorylated and/or active GPCRs. This property of $\beta$-arrestin2 is attributed to a more disordered C-domain, as compared to other arrestins. Specifically, $\beta$-sheet XIV (located in the C-domain $\beta$ sandwich) appears to be shortened, in comparison to $\beta$-arrestin1, and continues as an unstructured loop. As this structural component also takes part in interactions of the arrestin hinge region, close to the polar core, structural instability could lead to the spontaneous activation of $\beta$-arrestin2, without the need to engage phosphorylated intracellular domains of GPCRs. Thus, the authors of Zhan et al. ${ }^{39}$ provide an adequate explanation of why $\beta$-arrestin2 might be better suited to form phosphorylationindependent GPCR-complexes. Notably, this hypothesis has also been tested and supported by modelling ${ }^{40}$.

Strikingly, we were able to show that certain GPCRs are readily being regulated by overexpressed GRKs in a cellular system without ligand addition. Although ligand-independent GPCR phosphorylation has been described for multiple receptors ${ }^{36,41-44}$, it has not been convincingly shown to this point that this phosphorylation would translate into arrestin functions in a cellular context. We demonstrated that both, the V2R and AT1R couple to arrestins and internalise in a ligand-independent fashion as long as the essential GRKs are present at high expression levels (Figs. 4-6). Although GRK5 and 6 mediate this $\beta$-arrestin pre-coupling for both receptors, this is not a unique feature of the membrane-associated GRK4 family kinases. As GRK2 and 3 can achieve similar effects for the V2R, this rather has to be a distinct characteristic of a specific GPCR. Furthermore, the significantly increased basal molecular interaction between $\beta$-arrestin 2 and the respective receptor upon GRK overexpression was not abolished by pre-treatment with an inverse agonist or antagonist (Losartan ${ }^{45}$ was used for the AT1R and Tolvaptan ${ }^{46}$ for the V2R, Supplementary Figure 13). Hence, 
this specific pre-coupling effect is unlikely due to the constitutive activity of the respective GPCR. Especially, since this pre-coupling effect could not be transferred to the b2AR by exchange with the V2R C-terminus (Supplementary Figure 5b, c, m; 6), we can also exclude the C-terminus as sole mediator of GRK-specific processes.

Currently, it is unknown whether a single receptor is phosphorylated by a single kinase or by multiple kinases in a sequential manner. This could lead to vastly different outcomes, as we were able to show that different GRKs and second messenger kinases are able to induce divergent regulatory processes, depending on the targeted GPCR. Notably, the effect of PKC on the regulation of GRKs was described multiple times in literature $47-49$, as it was shown that the activity of GRKs can be either increased or decreased via PKC activity, depending on the used system. Even though we were able to record a reduction in GRK5- and 6-mediated $\beta$-arrestin recruitment to the AT1R under inhibition of PKC (Fig. 5e, f), we could not draw a conclusion on how PKC modulates the activity of specific kinases. The elucidation of these intriguing effects requires more in-depth analysis and possibly single-molecule studies.

Using our triple GRK KO cell lines, it became evident that different GPCRs require certain levels of GRK expression in order to recruit arrestins. In contrast to the b2AR (Fig. 1c), the PTH1R and M5R showed robust $\beta$-arrestin2 recruitment in $\Delta$ GRK3/5/6 cells (Supplementary Fig. 7a, b). This does not reflect on the ability of GRK2 to regulate the b2AR, as has been shown multiple times in literature and our presented overexpression experiments (Fig. 1e). These results rather demonstrate that the affinities of GRK isoforms to GPCRs differ depending on the individual receptor. Thus, the tissue-specific expression levels ${ }^{50}$ of individual GRKs in combination with their affinities to or formed complex configurations ${ }^{51}$ with specific receptors determine GPCR regulation.

Several studies have demonstrated phosphorylation of the b2AR at different serine and/or threonine residues ${ }^{43,52}$ and phosphorylation by GRK2 or 6 was shown to serve different functions ${ }^{8}$. In our study, we observed that all GRKs can mediate receptor $-\beta$-arrestin interactions to the same extent and by using a single $\beta$-arrestin2 conformational change sensor (FlAsH5 according to Nuber et $a{ }^{28}{ }^{28}$ ), we could demonstrate that the $\mathrm{N}$-domain of $\beta$-arrestin2, which recognises phosphorylated intracellular receptor domains, showed similar conformational changes for the different kinases (Fig. 2a). However, more experiments have to be performed to rule out that differential b2AR phosphorylation by GRK2 or 6 might lead to distinct $\beta$ arrestin 2 conformational changes. While different GRK isoforms might preferably phosphorylate distinct sites of the b2AR, resulting in different phosphorylation patterns of the C-terminus or IL3, our experiments clarify that the phosphorylation by each GRK isoform is sufficient to induce high-affinity $\beta$-arrestin recruitment (Fig. 1e).

Interactions between arrestins and the M2R were investigated previously ${ }^{53-55}$. Interestingly, the $\beta$-arrestin recruitment assay only induced minimal BRET changes for the M2R at endogenous GRK expression levels (Supplementary Fig. 14). However, upon overexpression of GRK2 or 3 robust $\beta$-arrestin recruitment was observed. It is tempting to speculate that the M2R exhibits a rather low affinity for GRKs to prevent its desensitisation since its function is essential for the reduction of heart rate ${ }^{56}$. Under pathophysiological conditions of GRK2 overexpression during chronic heart failure ${ }^{57}$, the M2R might internalise which could possibly contribute to tachycardiac effects in patients.

For each receptor case with unclear GRK assignment by statistical analysis (Fig. 3i, j), alternative kinases were previously reported to be involved in receptor phosphorylation. In the case of the M1R and M3R, casein kinase 1 alpha and casein kinase 2 were shown to be involved in receptor phosphorylation, respectively ${ }^{58,59}$. For the C5aR1, PKC $\beta$ was shown to contribute to receptor phosphorylation ${ }^{60}$. Therefore, our cellular platform for arrestin recruitment might be able to rapidly differentiate between receptors with purely GRK-dependent arrestin recruitment and receptors that rely on the action of other intracellular kinases for efficient arrestin binding, desensitisation and internalisation.

Using our $\triangle$ Q-GRK cell line, we were able to show that high expression levels of GRK2 and 3 are able to mediate $\beta$-arrestin interactions with all tested GPCRs. GRK5 and 6 seem to fulfil divergent roles depending on the analysed GPCR, as they were not able to induce $\beta$-arrestin-coupling to the M2R, M4R, M5R and MOP (Fig. 3i, j; Supplementary Fig. 5). Interestingly, this is not necessarily due to a lack of receptor phosphorylation, as we were able to show that GRK5 and 6 phosphorylate the MOP upon agonist activation, but fail to mediate $\beta$-arrestin recruitment and receptor internalisation (Supplementary Fig. 2). Nevertheless, we found receptors for which the GRK5- and 6-facilitated receptor regulation is indistinguishable from that mediated by GRK2 and 3 (namely the PTH1R and b2AR, Figs. 1e; 3c, d).

In an endeavour to match the measured GRK-specific $\beta$ arrestin recruitment with the main features of the tested GPCRs, we analysed the length of the respective C-terminus and IL3, as well as the number and relative location of their putative phosphorylation sites (Fig. 7, Supplementary Table 2). Here we were not able to find correlations that would compellingly explain the observed GRK-selectivity for our panel of GPCRs (Fig. 7a). Interestingly, none of the analysed class B receptors are solely regulated by GRK2 and 3, but all are regulated by GRK2/3/5/6 (b2V2 was excluded from this analysis as an unphysiological chimaera; Fig. 7b).

Further, we assessed the abundance and relative positions of previously established phosphorylation motifs ${ }^{61}$ (PPP, PXPP, PXPXXP, PXXPXXP, whereby P represents either Ser, Thr, Asp or Glu residues and X any amino acid), reported to be important for $\beta$-arrestin recruitment ${ }^{62,63}$. As illustrated in Fig. $7 \mathrm{c}-\mathrm{n}$, all motifs show a similar distribution between our defined GRKselectivity groups.

Interestingly, the PXPP motif showed a higher abundance in the central area $(0.25-0.75)$ of analysed peptide stretches of GPCRs regulated by GRK2/3. In contrast, the same motif was found more often in the peripheral area $(0.00-0.25$ and 0.75-1.00) of peptide stretches of GPCRs regulated by GRK2/3/5/ 6 (Fig. 7h). We found a significant association (Fisher's exact test, $p=0.0005)$ between the position of putative phosphorylation motifs (PXPP: central vs. peripheral) and GRK-specificity of the assessed GPCRs, according to our analysis. However, the causality of this association remains to be explained. Notably, although class A and B GPCRs are differentially represented in the GRKspecificity groups as defined in Fig. 3i, j, no significant association was found between the positions of PXPP motifs and the A-B classification (Fig. 7l; Fisher's exact test, $p=0.7328$ ). In general, we did not identify common features of C-terminal and IL3 sequences which would allow for the reliable prediction of GRK-selectivity. This again underlines the complexity of the GPCR regulatory system, as we were not able to link the existence or positioning of phosphorylation patterns that are suitable for the recruitment of $\beta$-arrestins with the observed GRK selectivity. Hence, we propose that GRK-selectivity is rather defined by the overall geometry of each GPCR and influenced not only by the availability of putative phosphorylation sites but also by the general promiscuity of other intracellular domains.

Recently, it has been reported that phosphorylation patterns, which promote $\beta$-arrestin recruitment, do not coincide with phosphorylation sites promoting $\beta$-arrestin activation ${ }^{64}$. Hence, it is still possible that the herein identified GRK-selectivity for $\beta$ arrestin recruitment might differ from GRK-selectivity toward 


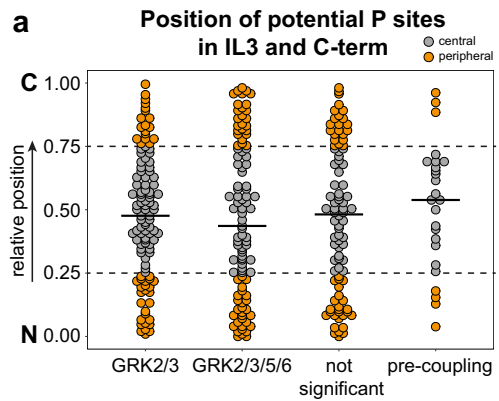

C

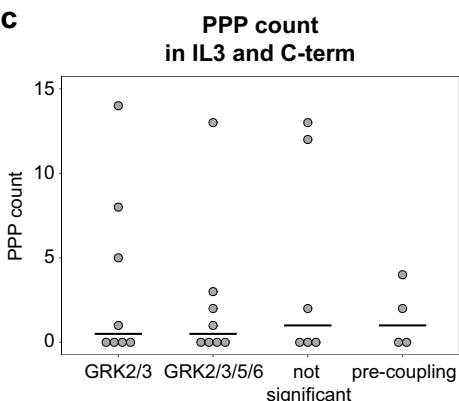

d
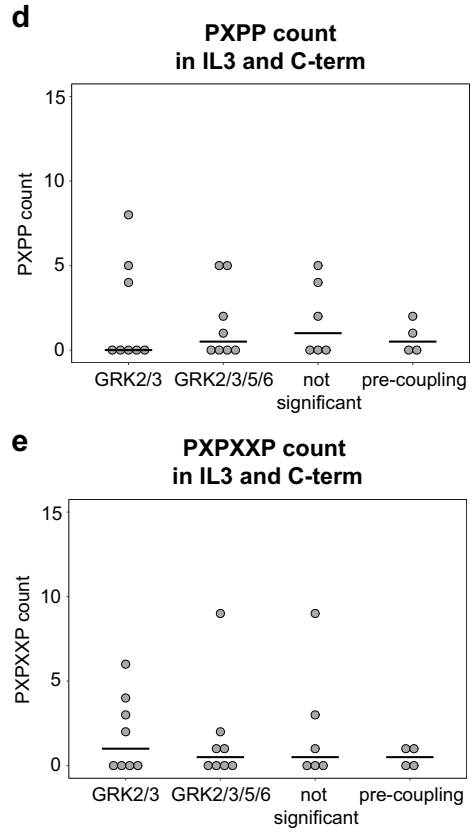

f

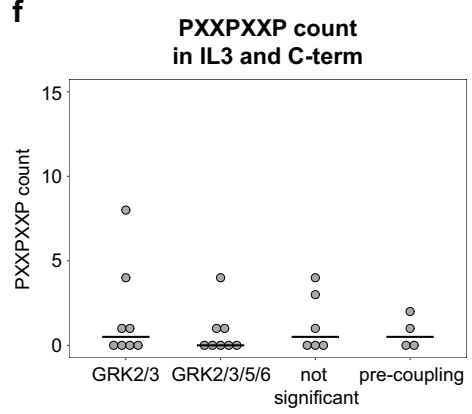

b
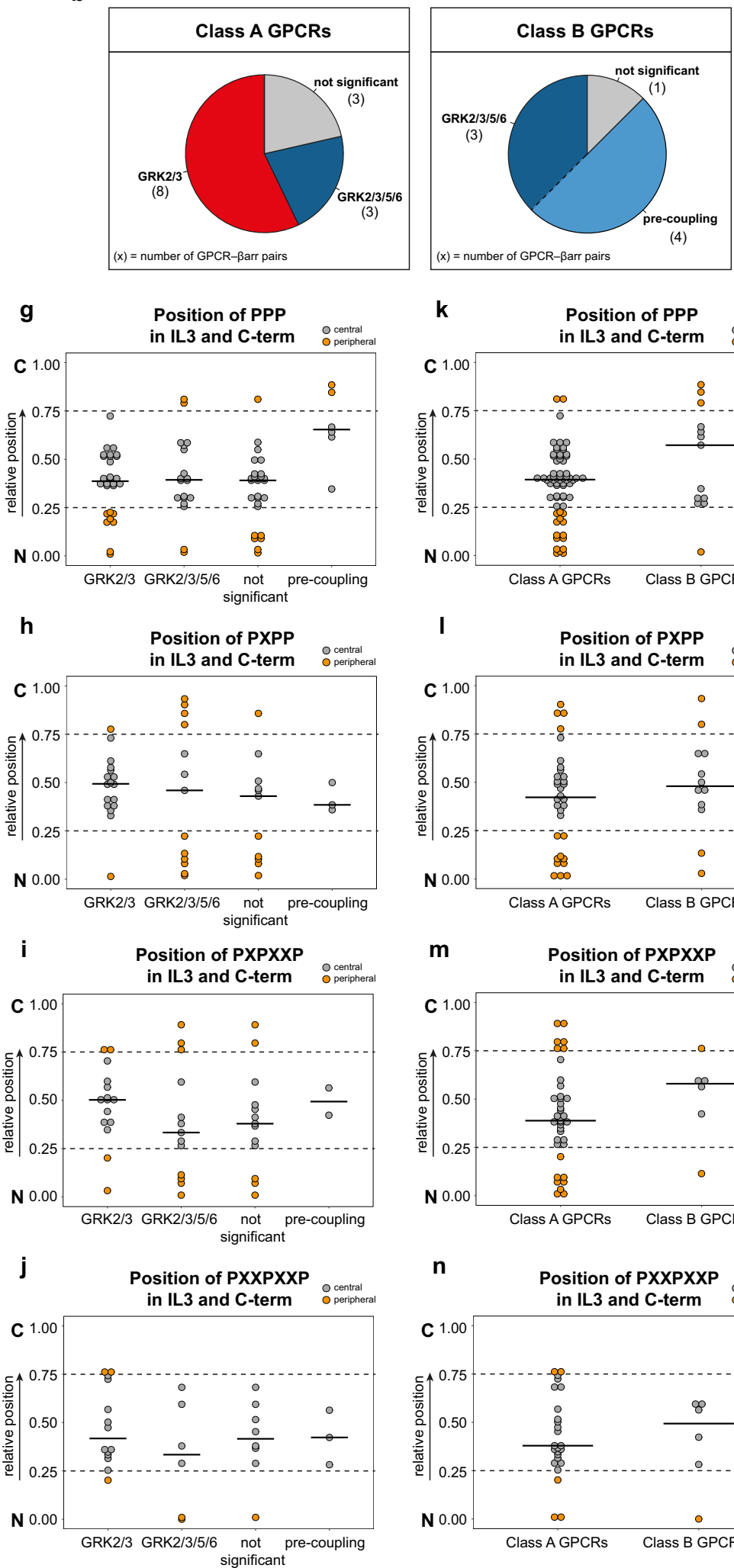
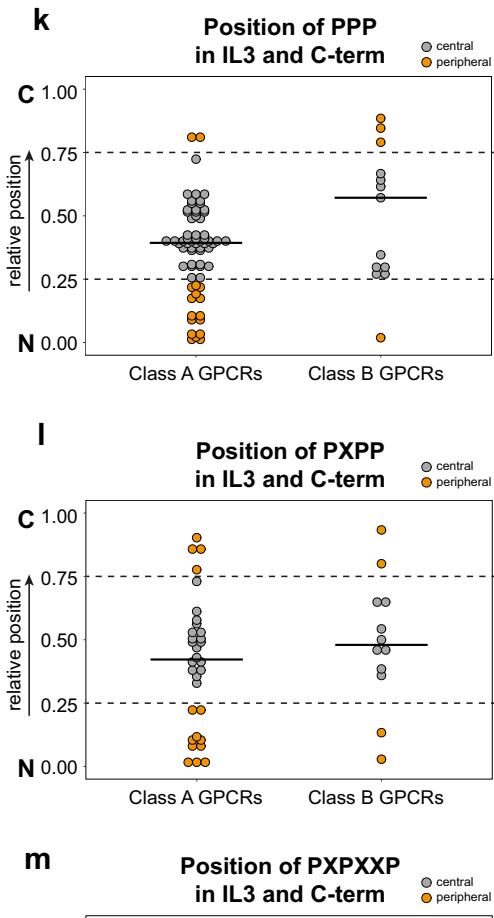

C 1.00
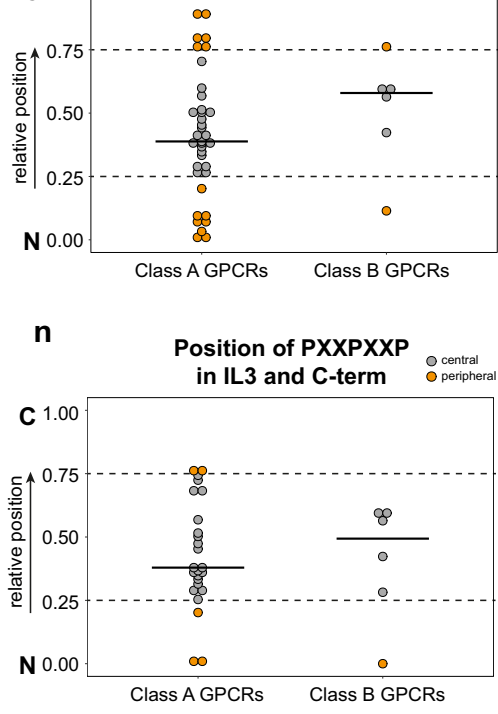

$\beta$-arrestin activation. More work has to be done to connect functional $\beta$-arrestin conformational changes with GRK-specific GPCR phosphorylation patterns to unravel those fine details of the phosphorylation barcode hypothesis.

Apart from phosphorylation patterns, Mayer et al ${ }^{65}$ proposed phosphorylation sites with specific functions based on conformational changes in arrestin-1 induced by binding to phosphopeptides. Intriguingly, the existence of inhibitory sites, which prevent arrestin binding, were predicted for several GPCRs including the AT1R. Since GRK5 and GRK6 overexpression abolished concentration-dependent $\beta$-arrestin recruitment to AT1R (Fig. 5e, f; Supplementary Fig. 11g, h), we created AT1R constructs lacking one of each inhibitory site $\left(A T 1 R^{S 347 A}\right.$ and $\left.\mathrm{AT}_{1} \mathrm{R}^{\mathrm{S} 348 \mathrm{~A}}\right)$ or both $\left(\mathrm{AT} 1 \mathrm{R}^{\mathrm{S} 347 \mathrm{~A} / \mathrm{S} 348 \mathrm{~A}}\right.$; Supplementary Fig. 15). 
Fig. 7 Putative phosphorylation motifs in IL3 and C-terminus of GPCRs are not associated with GRK-specific $\beta$-arrestin recruitment. Analysis of the abundance and position of identified putative serine and threonine (Ser/Thr) phosphorylation sites, clusters ${ }^{62}$ (PPP, PXPP) and patterns (PXXPXXP, PXPXXP, as identified by Zhou et al. ${ }^{61}$ ) in the intracellular loop 3 (IL3) and C-terminus (C-term; information from GPCRdb.org) of GPCRs listed in Supplementary Table 2 with the exception of the unphysiological b2V2 chimaera receptor. While $\mathrm{X}$ represents any amino acid, P may be a Ser, Thr or a negatively charged amino acid (glutamic acid, Glu; aspartic acid, Asp). The number and positions of potential phosphorylation sites, clusters and patterns were detected using Python 3.8.7. In order to compare positions of potential phosphorylation sites or motifs between GPCRs with varying lengths of IL3 and C-term, their relative position was calculated as the position index in relation to the full length of the respective peptide stretch. Consequently, the relative position of 0 corresponds to the beginning $(\mathrm{N})$, whereas a relative position of 1 corresponds to the end (C) of the respective peptide stretch. All relative positions are displayed as dot plots with the corresponding median, indicated by bars. Positions between 0.25 and 0.75 were categorised as central (grey) while positions between 0.0 and 0.25 as well as 0.75 and 1.00 were considered peripheral (orange). Dashed lines mark 0.25 and 0.75 breakpoints. a Relative position of potential phosphorylation sites (Ser/Thr) in the IL3 and C-term of analysed GPCRs grouped according to their GRK-specific $\beta$-arrestin ( $\beta$ arr) recruitment. In addition to the three groups defined in Fig. 3i, j (GRK2/3-regulated, GRK2/3/5/6-regulated and not significant), GPCRs displaying Barr pre-coupling (AT1R and V2R) were compiled in a fourth group (pre-coupling). $\mathbf{b}$ The analysed receptors were grouped as class A or B according to Oakley et al. ${ }^{62}$ (Supplementary Table 2) and each class is represented as one pie chart. In each pie chart, all GPCR- $\beta$ arr pairs are assigned to the different GRK-specific $\beta$ arr recruitment groups. The absolute number of GPCR- $\beta$ arr pairs presented in each group is indicated in brackets. Since GPCRs displaying $\beta$ arr pre-coupling can be considered a subgroup of GRK2/3/5/6-regulated GPCRs, both slices are separated by a dashed line (right pie). c-f Abundance of PPP clusters (c), PXPP clusters (d), PXPXXP patterns (e) and PXXPXXP patterns (f) are displayed as dot plots grouped according to their GRK-specific $\beta$ arr recruitment. g-n Relative positions of P-patterns as indicated. They were grouped according to their GRK-specific $\beta$ arr recruitment ( $\mathbf{g}$-j), as well as the GPCR classification ( $\mathbf{k}-\mathbf{n})$. Association between the position of PXPP and the GRK-specific Barr recruitment $(\mathbf{h}, p=0.0005)$ or GPCR classification $(\mathbf{I}, p=0.7328)$ was tested using Fisher's exact test. Exact odds ratios and confidence intervals are provided in the Source Data files.

Neither of the three mutant receptors showed an altered $\beta$ arrestin2 pre-coupling upon GRK5 or 6 overexpression (Supplementary Fig. $15 \mathrm{f}-\mathrm{m})$, yet alanine substitution of the $\mathrm{S}^{347}$ phosphorylation site reduced GRK2- and 3-specific $\beta$-arrestin2 recruitment to the level of $\mathrm{PKC}$-mediated $\beta$-arrestin 2 recruitment (Supplementary Fig. 15c). Thus, these two phosphorylation sites do not exhibit an inhibitory effect on $\beta$-arrestin 2 recruitment. Although the effect on $\beta$-arrestin1 recruitment remains to be examined, our results highlight the difference between the cellular functionality of arrestins and their behaviour towards synthesised phosphopeptides. Hence, it is imperative that future studies take all interaction interfaces between GPCRs and $\beta$-arrestin into account, as our analysis shows that there are more determinants for $\beta$-arrestin functions besides receptor phosphorylation at the C-terminus or IL3.

While GRK5 and 6 are membrane-localised ${ }^{66}$, GRK2 and 3 are primarily cytosolic and translocate to the plasma membrane supported by interactions with $\beta \gamma$-subunits of activated $G$ proteins ${ }^{67}$. It is still conceivable that GRK5 and 6 interactions with certain GPCRs might be obstructed due to their distinct cellular localisation. The plasma membrane features a rather heterogeneous distribution of proteins and it has been shown that certain GPCRs tend to reside in specific membranous microdomains ${ }^{68}$. Some receptors might localise in membranous compartments that are inaccessible for GRK5 and 6. Following this hypothesis, these GPCRs would be accessible to GRK2 and 3 since they emerge from the cytosol and would not be limited to two-dimensional diffusion and hindered by possible confinements. This still does not exclude the existence of GPCRs which do not serve as substrates for GRK2 or 3, due to e.g. low affinity.

While this manuscript was under revision, another group published three independently created $\triangle \mathrm{GRK}$ knockout cell lines $(\Delta \mathrm{GRK} 2 / 3, \Delta \mathrm{GRK} 5 / 6 \text { and } \Delta \mathrm{GRK} 2 / 3 / 5 / 6)^{69}$, which correspond to our GRK family knockout cell lines and $\Delta$ Q-GRK. The only common receptor among both manuscripts is the C5aR1. Similar to our findings, they showed a stronger loss of $\beta$-arrestin 1 recruitment to the C5aR1 in the absence of GRKs, as compared to $\beta$-arrestin2. Interestingly, using their family knockout cell lines, the authors found a very mild loss of arrestin recruitment for the knockout of GRK2 and 3, while the loss of GRK5 and 6 almost resembled the $\Delta \mathrm{GRK} 2 / 3 / 5 / 6$ condition. This would imply that the C5aR1 is mainly phosphorylated by GRK5 and 6. In contrast, our overexpression experiments suggest that all four GRKs are able to facilitate $\beta$-arrestin recruitment to a similar extent. Only using endogenous expression levels to investigate the influence of GRKs on $\beta$-arrestin recruitment could be misleading in this context, as this is strongly dependent on the utilised cell line. This is exemplified by the data shown in Fig. 1c, e. Here, we also determined GRK6 as the main driver of arrestin recruitment to the b2AR under endogenous expression levels of GRKs in HEK293 cells, while all GRKs are able to equally mediate this interaction when overexpressed. This strengthens the argument that GRK expression levels strongly influence the GRK-specificity of $\beta$-arrestin recruitment ${ }^{50}$.

In conclusion, we were able to elucidate the GRK-specificity of receptor regulation for 12 different GPCRs. Our analysis demonstrates that different GRK isoforms may have identical, overlapping or divergent functions, depending on the targeted GPCR. This adds another layer of complexity to the regulation of GPCR signalling and trafficking and a possible explanation of how different $\beta$-arrestin functions are mediated across various tissues and cell types, especially considering often dysregulated, pathophysiological GRK expression levels.

\section{Methods}

Cell culture. HEK293 cells were originally obtained from DSMZ Germany (ACC 305) and cultured in Dulbecco's modified Eagle's medium (DMEM; Sigma-Aldrich D6429), complemented with 10\% foetal calf serum (Sigma-Aldrich F7524) and 1\% of penicillin and streptomycin mixture (Sigma-Aldrich P0781) at $37^{\circ} \mathrm{C}$ with $5 \% \mathrm{CO}_{2}$. The cells were passaged every 3-4 days. Cells were regularly checked for mycoplasma infections using the LONZA MycoAlert mycoplasma detection kit (LT07-318).

CRISPR/Cas 9 mediated knockout of GRK2, 3, 5 and 6. Stable GRK knockout cells were generated by transient transfection using self-made PEI reagent (SigmaAldrich, 408727, diluted to $10 \mu \mathrm{g} / \mathrm{ml}, \mathrm{pH} 7.2$, adjusted with $\mathrm{HCl}$ ) of the parental cells (HEK293) with lentiCRISPR v2 plasmid ${ }^{70}$ (Addgene \#52961) containing target-specific gRNAs listed in Supplementary Table 5. Complementary forward and reverse oligos were annealed and ligated into the BsmBI-restricted lentiCRISPR v2 vector. This vector could also be used for the generation of viral particles, but in our approach, they were directly transfected into the target cells. In order to prevent side effects caused by multiple transfections and selection rounds, all cell clones were created in singular attempts. To knockout one specific GRK, four different gRNA constructs were simultaneously transfected. Inline, double, triple or quadruple knockout cells were generated by transfection of 8,12 or 16 respective gRNA constructs at once. The transfected cells were then selected using $1 \mu \mathrm{g} / \mathrm{ml}$ puromycin (Sigma-Aldrich \#P8833). Limited dilution was used to establish single-cell clones, which were then analysed for the absence of the target protein by Western blot analysis. A puromycin selected cell pool transfected with empty lentiCRISPR v2 plasmid was used as Control. 
Establishment of GRK expression constructs. The used pcDNA3-GRK2 expression construct was described before ${ }^{71}$. GRK3 (NCBI reference sequence NM_005160.4), GRK5 (NCBI reference sequence NM_005308.3) and GRK6 (NCBI reference sequence NM_001004106.3) were amplified by PCR using GRK-specific primers including restriction sites for HindIII (forward primer) and BamHI (reverse primer) (GRK3: Forward primer (fw)-CTT AAG CTT GCC ACC ATG GCG GAC CTG GAG GCTG, Reverse Primer (rev)-CTT AGG ATC CTA GAG GCC GTT GCT GTT TCTG; GRK5: fw-CTT AAG CTT GCC ACC ATG GAG CTG GAA AAC ATC GTG, rev-CTT AGG ATC CTA GCT GCT TCC GGT GGAG; GRK6: fw-CTT AAG CTT GCC ACC ATG GAG CTC GAG AAC ATC GTAG, rev-CTT AGG ATC CTA GAG GCG GGT GGG GAGC). GRK3 and 6 were amplified from human leucocyte cDNA, GRK5 was amplified from a betagalactosidase fusion plasmid described before ${ }^{72}$. The fragments were ligated into pcDNA3 plasmids after BamHI and HindIII digest. Sequences of all plasmids were validated by sequencing.

The kinase-dead (KD) mutants of GRK2, GRK5 and 6 were created by sitedirected mutagenesis resulting in GRK2-K220R, GRK5-K215R and GRK6-K215R.

Western blot. Cells were washed once with ice-cold PBS and subsequently lysed with RIPA Buffer (1\% NP-40, $1 \mathrm{mM}$ EDTA, $50 \mathrm{mM}$ Tris pH 7.4, $150 \mathrm{mM} \mathrm{NaCl}$ $0.25 \%$ sodium deoxycholate), supplemented with protease and phosphatase inhibitor cocktails (Roche, \#04693132001, \#04906845001). Cleared lysates were boiled with sodium dodecyl sulfate (SDS) loading buffer and $15 \mu \mathrm{g}$ of total protein were loaded onto each lane of $10 \%$ polyacrylamide gels. After transfer onto nitrocellulose membranes, the total protein was detected by using specific antibodies (GRK2: Santa Cruz sc-13143 (1:500); GRK3: Cell signalling technology \#80362 (1:250); GRK5: Santa Cruz, sc-518005 (1:250); GRK6: Cell signalling technology \#5878 (1:1000), Vinculin: Biozol BZL03106 (1:1000): Actin: Sigma-Aldrich, A5441 $(1: 2000))$. As secondary antibodies, we used SeraCare peroxidase-conjugated, Goat anti-rabbit (No. 5220-0336) and Goat anti-mouse (No. 5220-0341), 1:10,000.

For experiments using the MOP, phospho-specific antibodies see below. Quantification of the blots was done using Fujifilm Multi Gauge Software (V3.0).

Relative quantification of endogenous GRK expression in Control cells was performed as described elsewhere ${ }^{27}$. In brief, $\Delta \mathrm{Q}$-GRK cells were transfected with N-terminally HA-tagged GRK2, 3, 5 and 6 constructs and lysed after $24 \mathrm{~h}$. Protein standards of equal amounts of HA-tagged GRKs were prepared and loaded onto the same gel with three different lysates from Control cells. Three independent blots of the same lysates and standards were prepared and probed with the GRK specific antibodies. After quantification, the relative protein abundance was calculated in relation to the signal of the respective standard. The GRK3 signal detected by the GRK2 antibody and GRK5 signal detected by the GRK6 antibody was not considered.

Cell viability assay and determination of growth rates. Cells were seeded with a density of 5000 cells $/ 100 \mu$ in 96 -well plates, with $12-16$ wells per cell line. After $48 \mathrm{~h}$ of incubation at $37^{\circ} \mathrm{C}$ and $5 \% \mathrm{CO}_{2}, 20 \mu \mathrm{l}$ of the cell titre blue reagent (Promega, G8081) were added to 3-4 wells for each cell line, and the cells were further incubated for $1.5 \mathrm{~h}$ under the same growth conditions. After the incubation time, fluorescence (excitation $540 \mathrm{~nm}$, emission $610 \mathrm{~nm}$, Gain 40, top reading) was measured using a TECAN Infinite 200 (Tecan, Crailsheim, Germany) plate reader. The plate was further incubated and 72, 96 and $120 \mathrm{~h}$ after seeding, three wells were measured as just described. The proliferation was reported as a relative fluorescence signal compared to the first measurement ( $48 \mathrm{~h}$ after seeding) \pm standard error of the mean (SEM) of at least $n=3$ experiments. Using these data, the growth rates were determined for the three $24 \mathrm{~h}$ intervals and mean values \pm SEM are reported.

Stable MOP-expressing cells and immunoprecipitation experiments. A retroviral expression vector was created by replacing the mCherry expression cassette of pMSCV-IRES-mCherry FP (a gift from Dario Vignali, Addgene plasmid \#52114) with a neomycin resistance cassette of pcDNA3 plasmid resulting in an empty pMSCV-IRES-NEO vector. The open reading frame of $\mathrm{N}$-terminal haemagglutinin (HA) tagged murine MOP gene ${ }^{72}$ was inserted into the multi-cloning-site of this vector resulting in pMSCV-HA-MOP-IRES-NEO. Freshly produced retroviral particles were used to transduce GRK-KO cell clones or respective Control cells. The cells were selected with $1 \mathrm{mg} / \mathrm{ml}$ G418 (Gibco, 11811-031) for 10 days.

For immunoprecipitation experiments, $4 \times 10^{6}$ cells were seeded in a $21 \mathrm{~cm}^{2}$ dish and after $24 \mathrm{~h}$ stimulated for ten minutes with $10 \mu \mathrm{M}$ DAMGO ([D-Ala2, $\mathrm{N}$ MePhe4, Gly-ol]-enkephalin, Tocris 1171) in DMEM medium (Sigma-Aldrich, D6429) without supplements. After washing with ice-cold PBS, the cells were lysed in $500 \mu \mathrm{l}$ RIPA buffer as described in the "Western blot" section. Totally, $400 \mu \mathrm{g}$ of total protein lysates were incubated with $20 \mu \mathrm{l}$ of HA-beads slurry (Thermo Scientific, 26182) at $4^{\circ} \mathrm{C}$ on a turning wheel for $2 \mathrm{~h}$. The beads were then washed three times with RIPA buffer containing protease and phosphatase inhibitors and $75 \mu \mathrm{l}$ sample buffer ( $125 \mathrm{mM}$ Tris pH 6.8, 4\% SDS, $10 \%$ glycerol, $167 \mathrm{mM}$ DTT) was added and the samples were heated to $42^{\circ} \mathrm{C}$ for $20 \mathrm{~min}$. The samples were loaded onto $10 \%$ polyacrylamide gels $(7.5 \mu \mathrm{l}$ per 15 -well mini gel lane) and phosphorylation was detected using freshly prepared solution of rabbit polyclonal phosphosite-specific MOP antibodies ${ }^{22,23}$ (dilution for all 1:1000) anti-pT370 (7TM0319B), anti-pS375 (7TM0319C) anti-pT376 (7TM0319D) and anti-pT379
(7TM0319E), all obtained from 7TM Antibodies (Jena, Germany). The total receptors were detected with an anti-HA-antibody (Cell signalling technology \# 3724; 1:1000). Quantification of the blots was done using Fujifilm Multi Gauge Software (V3.0).

Intermolecular bioluminescence resonance energy transfer (BRET). The GRKselective $\beta$-arrestin recruitment assay was performed either in Control or specific $\Delta$ GRK cell lines. In $21 \mathrm{~cm}^{2}$ dishes, $1.6 \times 10^{6}$ cells were seeded and transfected the next day with $0.5 \mu \mathrm{g}$ of the respective GPCR C-terminally fused to Nano luciferase (NanoLuc), $1 \mu \mathrm{g}$ of $\beta$-arrestin constructs $\mathrm{N}$-terminally fused to a Halo-ligand binding Halo-Tag and $0.25 \mu \mathrm{g}$ of one GRK or empty vector. In the case of the PTH1R and V2R, the BRET pair was swapped. All transfections were conducted following the Effectene transfection reagent manual by Qiagen (\#301427) and then incubated at $37^{\circ} \mathrm{C}$ overnight. Into poly-D-lysine-coated 96-well plates (Brand, 781965), 40,000 cells were seeded per well in presence of Halo-ligand (Promega, G980A) at a ratio of 1:2000. A mock labelling condition without the addition of the Halo-ligand was seeded for each transfection. After $24 \mathrm{~h}$, the cells were washed twice with measuring buffer $(140 \mathrm{mM} \mathrm{NaCl}, 10 \mathrm{mM}$ HEPES, $5.4 \mathrm{mM} \mathrm{KCl}, 2 \mathrm{mM}$ $\mathrm{CaCl}_{2}, 1 \mathrm{mM} \mathrm{MgCl} ; \mathrm{pH} \mathrm{7.3)}$ and NanoLuc-substrate furimazine (Promega, N157B) was added in a ratio of 1:35,000 in measuring buffer. A Synergy Neo2 plate reader (Biotek), operated with the Gen5 software (version 2.09), with a custommade filter (excitation bandwidth 541-550 nm, emission 560-595 nm, fluorescence filter $620 / 15 \mathrm{~nm}$ ) was used to perform the measurements. The baseline was monitored for $3 \mathrm{~min}$. After the addition of the respective agonist, the measurements were continued for five minutes. By subtracting the values measured for mock labelling conditions, the initial BRET change was corrected for labelling efficiency. Halo-corrected BRET changes were calculated by the division of the corrected and averaged values measured after ligand stimulation by the respective, corrected and averaged baseline values. Subsequently, this corrected BRET change was divided by the vehicle control for the final dynamic $\Delta$ net BRET change. These calculations were conducted using Excel 2016.

For the analysis of the respective BRET ratios before and after stimulation, the Halo-corrected and averaged BRET ratios before stimulation (baseline) and the Halo-corrected and averaged BRET ratios after stimulation with saturating ligand concentration (stimulated) are displayed as bar graphs. $\mathrm{EC}_{50}$ values and the corresponding SEM for concentration-dependent $\beta$-arrestin recruitment were calculated with GraphPad Prism 7.03. using curves that were plotted from $n=3$ independent experiments.

Receptors were stimulated as follows: human angiotensin II type 1 receptor (AT1R) with angiotensin II (AngII; Tocris 4474-91-3, in measuring buffer), human $\beta 2$ adrenergic receptor (b2AR) with isoproterenol (Iso; Sigma-Aldrich I5627, in water) Epinephrine (Sigma-Aldrich E4642, in measuring buffer) and Norepinephrine (Sigma-Aldrich 74488, in measuring buffer), human b2AR with an exchanged C-terminus of the vasopressin type 2 receptor (b2V2) with Iso, human complement 5a receptor 1 (C5aR1) with C5aR-agonist (AnaSpec AS65121, in measuring buffer), human muscarinic 1-5 acetylcholine receptors (M1R, M2R, M3R, M4R and M5R) with acetylcholine (ACh; Sigma-Aldrich A6625, in measuring buffer), murine MOP with [D- $\mathrm{Ala}^{2}, \mathrm{~N}$-MePhe ${ }^{4}$, Gly-ol]-enkephalin (DAMGO; Tocris 1171, in water), human parathyroid hormone 1 receptor (PTH1R) with parathyroid hormone (1-34) (PTH(1-34); Bachem 4011474, in measuring buffer) and human vasopressin type 2 receptor (V2R) with $\left[\mathrm{Arg}^{8}\right]$ vasopressin (AVP; Tocris 2935, in water). The transfected $\beta$-arrestins are of bovine origin. $\beta$-arrestinl constructs lacking the finger loop region (dFLR) were generated as described in Cahill et al. ${ }^{32}$ by site-directed mutagenesis. The corresponding $\beta$ arrestin 2 constructs were designed homologously.

If not further elaborated, the utilised cDNAs were obtained from the cDNA resource centre (www.cDNA.org) or Addgene. The Halo-Tag or NanoLuc genes were acquired from Promega and were genetically fused to the respective N- or C-termini.

In the case of cmpd101 (Tocris 15777006, in DMSO) and pindolol (SigmaAldrich, $\mathrm{P} 0778$, in $0.1 \mathrm{M} \mathrm{HCl}$ ) inhibitor experiments, the $\beta$-arrestin recruitment was induced with either $1 \mu \mathrm{M}$ isoproterenol (in case of the b2AR) or $10 \mu \mathrm{M} \mathrm{ACh}$ (in case of the M2R) after a 10-min incubation period with different concentrations of cmpd101 or pindolol.

In the case of the Gö6983 (Tocris, 2285, in DMSO) experiments, the cells were pre-incubated with $500 \mathrm{nM}$ of the inhibitor at $37^{\circ} \mathrm{C}$ for $1 \mathrm{~h}$ and subsequently stimulated with different concentrations of AngII.

For the inverse agonist or antagonist experiments conducted for the V2R and AT1R, the cells were treated with the indicated concentration of Tolvaptan $(1 \mu \mathrm{M})$ or Losartan $(10 \mu \mathrm{M}) 4 \mathrm{~h}$ after the transfer into 96-well plates and incubated overnight. The following washing steps, as well as the experimental procedure, were carried out with buffers containing the same concentration of the inverse agonist or antagonist, to guarantee unchanged receptor occupancy by the compounds. After the acquisition of baseline BRET ratios, the cells were stimulated with $1 \mu \mathrm{M}$ AVP or $1 \mu \mathrm{M}$ AngII, respectively. Control conditions without inverse agonist or antagonist treatment were measured again, side-by-side, to enable data comparability.

Fluorometric assessment of GRK expression and functionality of GRK-YFP constructs. For the assessment of GRK expression levels, C-terminal GRK-YFP fusions were constructed via isothermal plasmid assembly, keeping the identical 
vector backbone. $1.6 \times 10^{6} \Delta \mathrm{Q}$-GRK cells were seeded in $21 \mathrm{~cm}^{2}$ dishes and transfected the next day with $1.5 \mu \mathrm{g}$ of either GRK2-, 3-, 5- or 6-YFP fusion constructs. All transfections were conducted following the Effectene transfection reagent manual by Qiagen $(\# 301427)$ and then incubated at $37^{\circ} \mathrm{C}$ overnight. Into poly-D-lysine-coated 96-well plates (Brand, 781965), 40,000 cells were seeded per well. After $24 \mathrm{~h}$ of incubation at $37^{\circ} \mathrm{C}$, the fluorescence was assessed using a Synergy Neo2 plate reader (Biotek) and a corresponding YFP filter (excitation bandwidth $465-505 \mathrm{~nm}$, emission $496-536 \mathrm{~nm}$ ). To confirm the catalytic activity of the used GRK-YFP fusion constructs, GRK-specific $\beta$-arrestin2 recruitment intermolecular BRET assay featuring the PTH1R-Halo-Tag and $\beta$-arrestin2NanoLuc was performed. In this case, cells were transfected as described in "intermolecular bioluminescence resonance energy transfer (BRET)" section. Instead of untagged GRK constructs, $0.25 \mu \mathrm{g}$ of the GRK-YFP fusion constructs were transfected. After stimulation with PTH(1-34), the data were recorded and processed as described above.

Statistical analysis of intermolecular BRET. BRET ratios and fold changes are displayed as the mean of at least three independent experiments with error bars indicating the SEM. Statistical analysis was performed using Student's $t$-test or analysis of variance (ANOVA; one-way or two-way mixed model ANOVA), as well as appropriate multiple comparisons as indicated in corresponding figure legends. Data were prepared using Python 3.8.7 and statistical analysis was conducted in $\mathrm{R}$ $4.0 .3^{73}$. A type I error probability of 0.05 was considered to be significant in all cases. Two-way mixed model ANOVA was performed using $e z \mathrm{R}$ package (Lawrence, MA. (2011) ez: Easy analysis and visualisation of factorial experiments. $\mathrm{R}$ package version 4.4-0. http://CRAN.R-project.org/package $=\mathrm{ez}$ ) and multiple comparisons were conducted using the multcomp $\mathrm{R}$ package ${ }^{74}$. The clustering heatmap was generated using the pheatmap R package (Kolde, R. (2013). pheatmap: Pretty Heatmaps. R package version 1.0.12. http://CRAN.R-project.org/ package $=$ pheatmap.). Additionally, all code that was created for the statistical analysis of presented data can be accessed via 10.5281/zenodo.5764249.

Intramolecular BRET. $\triangle \mathrm{Q}$-GRK or Control cells were transfected with $1.2 \mu \mathrm{g}$ untagged b2AR, $0.12 \mu \mathrm{g}$ of $\beta$-arrestin2 FlAsH5-tagged biosensor C-terminally coupled to NanoLuc, $0.25 \mu \mathrm{g}$ of either GRK2, 3, 5, 6 or empty vector as noted, following the Effectene transfection reagent protocol by Qiagen. $24 \mathrm{~h}$ after transfection, 40,000 cells were seeded per well into poly-D-lysine coated 96 -well plates and incubated overnight at $37^{\circ} \mathrm{C}$. For this study, the FlAsH (fluorescein arsenical hairpin-binder)-labelling procedure previously described Hoffmann et al. ${ }^{75}$ was adjusted for 96 -well plates. In brief, the cells were washed twice with PBS, then incubated with $250 \mathrm{nM}$ FlAsH in labelling buffer ( $150 \mathrm{mM} \mathrm{NaCl}, 10 \mathrm{mM}$ HEPES, $25 \mathrm{mM} \mathrm{KCl}, 4 \mathrm{mM} \mathrm{CaCl}_{2}, 2 \mathrm{mM}$ $\mathrm{MgCl}_{2}, 10 \mathrm{mM}$ glucose; $\mathrm{pH} 7.3$ ), complemented with $12.5 \mu \mathrm{M}$ 1,2-ethane dithiol (EDT) for sixty minutes at $37^{\circ} \mathrm{C}$. After aspiration of the FlAsH labelling or mock labelling solutions, the cells were incubated for $10 \mathrm{~min}$ at $37^{\circ} \mathrm{C}$ with $100 \mu \mathrm{l} 250 \mu \mathrm{M}$ EDT in labelling buffer per well. In addition to the NanoLuc substrate, measurement and analysis of the BRET change was performed as described above (see Section "Intermolecular bioluminescence resonance energy transfer (BRET)").

Microscopy. The morphology of the generated cell clones was documented during regular cell culture procedures using phase-contrast microscopy at the Invitrogen EVOS FL Auto in 10× magnification.

Receptor internalisation of fixed cells stably expressing the MOP was analysed using confocal microscopy. $\Delta$ GRK2/3, $\Delta$ GRK5/6, $\Delta$ Q-GRK and Control cells were grown on poly-L-lysine-coated coverslips for 2-3 days. After the treatment with $10 \mu \mathrm{M}$ DAMGO at $37^{\circ} \mathrm{C}$ for $30 \mathrm{~min}$, cells were fixed with $4 \%$ paraformaldehyde and $0.2 \%$ picric acid in phosphate buffer ( $\mathrm{pH} 6.9)$ for $30 \mathrm{~min}$ at room temperature. Then coverslips were washed several times with PBS w/o Ca ${ }^{2+} / \mathrm{Mg}^{2+}$ buffer. After washing with $50 \%$ and $100 \%$ methanol for $3 \mathrm{~min}$, cells were permeabilised with phosphate buffer for $2 \mathrm{~h}$ and then incubated with anti-HA antibody (7TM000HA, 7TM Antibodies (Jena, Germany), 1:500) followed by Alexa488-conjugated secondary antibody (1:2000) (Life Technologies, Thermo Fisher Scientific A11008). Specimens were mounted with Roti ${ }^{\circ}$-Mount FluorCare DAPI (Carl Roth, HP20.1) and examined using a Zeiss LSM510 META laser scanning confocal microscope.

Live cell experiments were performed to record the translocation of PTH1R, M5R, V2R or AT1R, $\beta$-arrestin and the early endosome marker Rab5 upon agonist stimulation at a Leica SP8 laser scanning confocal microscope operated with the Leica Application Suite X (version 3.5.5.19976). Therefore $\Delta$ GRK2/3, $\Delta$ GRK5/6, $\Delta$ Q-GRK and Control cells were transfected with $1 \mu \mathrm{g}$ of the C-terminally CFP-fused receptor (in the case of AT1R, we included an HSP-export tag ${ }^{76}$ and transfected $1.25 \mu \mathrm{g}$ ), $0.5 \mu \mathrm{g}$ of $\beta$-arrestin-YFP, $0.5 \mu \mathrm{g}$ of Rab5-mCherry (kindly provided by Tom Kirchhausen (Harvard Medical School, Boston, USA)) and $0.25 \mu \mathrm{g}$ GRK expression constructs (as indicated) in a $21 \mathrm{~cm}^{2}$ dish, according to the Effectene transfection reagent manual by Qiagen. After $24 \mathrm{~h}, 700,000$ transfected cells were seeded onto poly-D-lysine-coated glass coverslips in 6-well plates. Another $24 \mathrm{~h}$ later, the coverslips were washed twice with measuring buffer and subsequently imaged before and after stimulation for $15 \mathrm{~min}$ with either $100 \mathrm{nM}$ PTH(1-34), $100 \mu \mathrm{M} \mathrm{ACh}, 100 \mathrm{nM}$ AVP or $100 \mathrm{nM}$ AngII as indicated. CFP was excited at a wavelength of $442 \mathrm{~nm}$, YFP at $514 \mathrm{~nm}$ and mCherry at $561 \mathrm{~nm}$. The images were acquired with a $63 \times$ water immersion objective, with zoom factor 3 , line average 3 and $400 \mathrm{~Hz}$ in $1024 \times 1024$ pixel format. Subcellular features of the acquired images were segmented and quantified using an ImageJ-based software (Image version 1.52p) called segmentation and quantification of subcellular shapes (Squassh). Utilising Squassh's deconvolution, denoising and segmentation of the three fluorescence channels present in each image, the raw data readout was then eligible for analysis using the R-based software SquasshAnalyst as described by A. Rizk ${ }^{77,78}$. All image-derived data in this study were processed and analysed with this method and are presented as fold change in colocalisation signal. Statistical analysis of quantified microscopy data was performed using GraphPad Prism 7.03. Unstimulated and stimulated co-localisation was compared using paired t-test. To identify significantly increased co-localisation under unstimulated conditions, quantified co-localisation was compared using ANOVA and two-sided Dunnett's test.

Enzyme-linked immunosorbent assay (ELISA). Receptor internalisation was quantified using a linear surface receptor ELISA that has been characterised extensively 79,80 . Equal numbers of $\Delta \mathrm{GRK} 2 / 3, \Delta \mathrm{GRK} 5 / 6, \Delta \mathrm{Q}$-GRK and Control cells stably expressing HA-tagged murine MOP were seeded onto poly-L-lysinecoated 24-well plates for 2-3 days. Then, cells were pre-incubated with anti-HA antibody (7TM000HA), obtained from 7TM Antibodies (Jena, Germany, 1:500) for $2 \mathrm{~h}$ at $4{ }^{\circ} \mathrm{C}$. After $30 \mathrm{~min}$ treatment with $10 \mu \mathrm{M}$ DAMGO at $37^{\circ} \mathrm{C}$, the cells were fixed with $4 \%$ paraformaldehyde and $0.2 \%$ picric acid in phosphate buffer $(\mathrm{pH} 6.9)$ for $30 \mathrm{~min}$ at room temperature and incubated with peroxidase-conjugated antirabbit antibody (Cell Signalling technology \#7074, 1:1500) overnight at $4^{\circ} \mathrm{C}$. After washing, the plates were developed with ABTS solution (Sigma-Aldrich A3219) and analysed at $405 \mathrm{~nm}$ using a microplate reader.

Label-free DMR biosensing. Dynamic mass redistribution (DMR) experiments were performed using the Corning Epic (Corning, NY, USA) biosensor technology as previously described in detail ${ }^{38,81-86}$. In short, for DMR detection $9 \times 10^{5} \Delta \mathrm{Q}-$ GRK cells were seeded into $21 \mathrm{~cm}^{2}$ dishes and cultured until reaching a confluence of $60-80 \%$, which is critical for the maintenance of a consistent proliferation phenotype and for comparable transfection efficiencies. Subconfluent cells were then transiently transfected with empty vector (pcDNA3) or expression plasmids (pcDNA3-based) encoding for the AT1R (HSP-AT1R-CFP), $\beta$-arrestin2, GRK2 or GRK6. The next day, transfected cells were seeded at a density of $2 \times 10^{4}$ cells per well into Corning Epic biosensor microplates and incubated overnight $\left(37^{\circ} \mathrm{C}, 5 \%\right.$ $\mathrm{CO}_{2}$ ). Prior to DMR detection, cells were washed three times with HBSS buffer containing $20 \mathrm{mM}$ HEPES and $0.1 \%$ fatty acid-free bovine serum albumin (SigmaAldrich). Cells were then placed into the Epic-DMR-reader, operated with the software Epic Imager 2012 and equilibrated for $1 \mathrm{~h}$ at $37^{\circ} \mathrm{C}$ to achieve baseline stabilisation. Compounds diluted in the same buffer were added to the biosensor plate using the CyBio Selma semi-automatic pipettor (Analytik Jena AG) and ligand-induced DMR alterations were monitored as picometre $(\mathrm{pm})$ wavelength shifts for at least $3600 \mathrm{~s}$ in $15 \mathrm{~s}$ intervals. Real-time DMR recordings are means + SEM of three technical replicates and were corrected by the pm wavelength shifts obtained in empty vector transfectants. Concentration-effect curves are means \pm SEM of $n=3$ independent biological replicates and were derived from the area under the curve between zero and $1800 \mathrm{~s}$ using a three-parameter logistic equation and the GraphPad Prism (8.4.3) software. Curves were fitted with "bottom" constrained to zero while all other settings were left to their default values. Two way ANOVA was used for statistical analysis.

Cell line availability. All created cell lines will be made available upon request.

Reporting summary. Further information on research design is available in the Nature Research Reporting Summary linked to this article.

\section{Data availability}

All data supporting the findings of this study are available within the article and its supplementary information files. Additional information, relevant data and unique biological materials will be available from the corresponding author upon reasonable request. Source data are provided with this paper.

\section{Code availability}

Custom code was created for general statistical analysis as well as for the analyses presented in Fig. $3 \mathrm{i}$ and Fig. 7. The code is available via Github and under https://doi.org/ 10.5281 /zenodo. 5764248 .

Received: 17 June 2021; Accepted: 6 January 2022; Published online: 27 January 2022

\section{References}

1. Sriram, K. \& Insel, P. A. G protein-coupled receptors as targets for approved drugs: how many targets and how many drugs? Mol. Pharmacol. 93, 251-258 (2018). 
2. Wingler, L. M. \& Lefkowitz, R. J. Conformational basis of G protein-coupled receptor signaling versatility. Trends Cell Biol. 30, 736-747 (2020).

3. Premont, R. T. \& Gainetdinov, R. R. Physiological roles of G protein-coupled receptor kinases and arrestins. Annu. Rev. Physiol. 69, 511-534 (2007).

4. Milligan, G. \& Kostenis, E. Heterotrimeric G-proteins: a short history. Br. J. Pharmacol. 147, S46-S55 (2006).

5. Gurevich, E. V., Tesmer, J. J., Mushegian, A. \& Gurevich, V. V. G proteincoupled receptor kinases: more than just kinases and not only for GPCRs. Pharmacol. Ther. 133, 40-69 (2012).

6. Kenakin, T. Biased receptor signaling in drug discovery. Pharmacol. Rev. 71, 267-315 (2019).

7. Liggett, S. B. Phosphorylation barcoding as a mechanism of directing GPCR signaling. Sci. Signal. 4, pe36 (2011).

8. Nobles, K. N. et al. Distinct phosphorylation sites on the beta(2)-adrenergic receptor establish a barcode that encodes differential functions of betaarrestin. Sci. Signal. 4, ra51 (2011).

9. Tobin, A. B., Butcher, A. J. \& Kong, K. C. Location, location, location...sitespecific GPCR phosphorylation offers a mechanism for cell-type-specific signalling. Trends Pharmacol. Sci. 29, 413-420 (2008).

10. Violin, J. D. \& Lefkowitz, R. J. Beta-arrestin-biased ligands at seventransmembrane receptors. Trends Pharmacol. Sci. 28, 416-422 (2007).

11. Urban, J. D. et al. Functional selectivity and classical concepts of quantitative pharmacology. J. Pharmacol. Exp. Ther. 320, 1-13 (2007).

12. Peterson, Y. K. \& Luttrell, L. M. The diverse roles of arrestin scaffolds in G protein-coupled receptor signaling. Pharmacol. Rev. 69, 256-297 (2017).

13. Whalen, E. J., Rajagopal, S. \& Lefkowitz, R. J. Therapeutic potential of betaarrestin- and G protein-biased agonists. Trends Mol. Med. 17, 126-139 (2011).

14. Gurevich, V. V. \& Benovic, J. L. Visual arrestin interaction with rhodopsin Sequential multisite binding ensures strict selectivity toward light-activated phosphorylated rhodopsin. J. Biol. Chem. 268, 11628-11638 (1993).

15. Shukla, A. K. et al. Structure of active beta-arrestin-1 bound to a G-proteincoupled receptor phosphopeptide. Nature 497, 137-141 (2013).

16. Kang, Y. et al. Crystal structure of rhodopsin bound to arrestin by femtosecond X-ray laser. Nature 523, 561-567 (2015).

17. Thomsen, A. R. B. et al. GPCR-G protein-beta-arrestin super-complex mediates sustained G protein signaling. Cell 166, 907-919 (2016).

18. Mann, A. et al. Agonist-induced phosphorylation bar code and differential post-activation signaling of the delta opioid receptor revealed by phosphositespecific antibodies. Sci. Rep. 10, 8585 (2020).

19. Mann, A. et al. Agonist-selective NOP receptor phosphorylation correlates in vitro and in vivo and reveals differential post-activation signaling by chemically diverse agonists. Sci. Signal. 12, eaau8072 (2019).

20. Kim, J. et al. Functional antagonism of different $\mathrm{G}$ protein-coupled receptor kinases for beta-arrestin-mediated angiotensin II receptor signaling. Proc. Natl Acad. Sci. USA 102, 1442-1447 (2005).

21. Moller, T. C. et al. Dissecting the roles of GRK2 and GRK3 in mu-opioid receptor internalization and beta-arrestin2 recruitment using CRISPR/Cas9edited HEK293 cells. Sci. Rep. 10, 17395 (2020).

22. Doll, C. et al. Deciphering micro-opioid receptor phosphorylation and dephosphorylation in HEK293 cells. Br. J. Pharmacol. 167, 1259-1270 (2012).

23. Just, S. et al. Differentiation of opioid drug effects by hierarchical multi-site phosphorylation. Mol. Pharmacol. 83, 633-639 (2013).

24. Butcher, A. J. et al. Differential G-protein-coupled receptor phosphorylation provides evidence for a signaling bar code. J. Biol. Chem. 286, 11506-11518 (2011).

25. Mann, A., Illing, S., Miess, E. \& Schulz, S. Different mechanisms of homologous and heterologous mu-opioid receptor phosphorylation. Br. J. Pharmacol. 172, 311-316 (2015).

26. Feng, B., Li, Z. \& Wang, J. B. Protein kinase C-mediated phosphorylation of the mu-opioid receptor and its effects on receptor signaling. Mol. Pharmacol. 79, 768-775 (2011)

27. Reichel, M., Weitzel, V., Klement, L., Hoffmann, C. \& Drube, J. Suitability of GRK antibodies for individual detection and quantification of GRK isoforms in western blots. Int. J. Mol. Sci. (2022) in press.

28. Nuber, S. et al. beta-Arrestin biosensors reveal a rapid, receptor-dependent activation/deactivation cycle. Nature 531, 661-664 (2016).

29. Reiner, S., Ambrosio, M., Hoffmann, C. \& Lohse, M. J. Differential signaling of the endogenous agonists at the beta2-adrenergic receptor. J. Biol. Chem. 285, 36188-36198 (2010).

30. Heitzler, D. et al. Competing G protein-coupled receptor kinases balance G protein and beta-arrestin signaling. Mol. Syst. Biol. 8, 590 (2012).

31. Ren, X. R. et al. Different $G$ protein-coupled receptor kinases govern $G$ protein and beta-arrestin-mediated signaling of V2 vasopressin receptor. Proc. Natl Acad. Sci. USA 102, 1448-1453 (2005).

32. Cahill, T. J. 3rd et al. Distinct conformations of GPCR-beta-arrestin complexes mediate desensitization, signaling, and endocytosis. Proc. Natl Acad. Sci. USA 114, 2562-2567 (2017).
33. Snyder, J. C., Rochelle, L. K., Barak, L. S. \& Caron, M. G. The stem cellexpressed receptor Lgr5 possesses canonical and functionally active molecular determinants critical to beta-arrestin-2 recruitment. PLoS ONE 8, e84476 (2013).

34. Gagnon, L. et al. Genetic code expansion and photocross-linking identify different beta-arrestin binding modes to the angiotensin II type 1 receptor. $J$. Biol. Chem. 294, 17409-17420 (2019).

35. Toth, A. D. et al. Heterologous phosphorylation-induced formation of a stability lock permits regulation of inactive receptors by beta-arrestins. J. Biol. Chem. 293, 876-892 (2018).

36. Oppermann, M., Freedman, N. J., Alexander, R. W. \& Lefkowitz, R. J. Phosphorylation of the type 1A angiotensin II receptor by G protein-coupled receptor kinases and protein kinase C. J. Biol. Chem. 271, 13266-13272 (1996).

37. Eguchi, A. et al. GRK5 is a regulator of fibroblast activation and cardiac fibrosis. Proc. Natl Acad. Sci. USA 118, e2012854118 (2021).

38. Schroder, R. et al. Deconvolution of complex G protein-coupled receptor signaling in live cells using dynamic mass redistribution measurements. Nat. Biotechnol. 28, 943-949 (2010).

39. Zhan, X., Gimenez, L. E., Gurevich, V. V. \& Spiller, B. W. Crystal structure of arrestin-3 reveals the basis of the difference in receptor binding between two non-visual subtypes. J. Mol. Biol. 406, 467-478 (2011).

40. Sensoy, O., Moreira, I. S. \& Morra, G. Understanding the differential selectivity of arrestins toward the phosphorylation state of the receptor. ACS Chem. Neurosci. 7, 1212-1224 (2016).

41. Diviani, D. et al. Effect of different G protein-coupled receptor kinases on phosphorylation and desensitization of the alphalB-adrenergic receptor. J. Biol. Chem. 271, 5049-5058 (1996).

42. Li, L. et al. G protein-coupled receptor kinases of the GRK4 protein subfamily phosphorylate inactive G protein-coupled receptors (GPCRs). J. Biol. Chem. 290, 10775-10790 (2015)

43. Tran, T. M. et al. Characterization of agonist stimulation of cAMP-dependent protein kinase and G protein-coupled receptor kinase phosphorylation of the beta2-adrenergic receptor using phosphoserine-specific antibodies. Mol. Pharmacol. 65, 196-206 (2004).

44. Baameur, F. et al. Role for the regulator of G-protein signaling homology domain of $\mathrm{G}$ protein-coupled receptor kinases 5 and 6 in beta 2 -adrenergic receptor and rhodopsin phosphorylation. Mol. Pharmacol. 77, 405-415 (2010).

45. Takezako, T., Unal, H., Karnik, S. S. \& Node, K. Structure-function basis of attenuated inverse agonism of angiotensin II type 1 receptor blockers for active-state angiotensin II type 1 receptor. Mol. Pharmacol. 88, 488-501 (2015).

46. Yamamura, Y. et al. OPC-41061, a highly potent human vasopressin V2-receptor antagonist: pharmacological profile and aquaretic effect by single and multiple oral dosing in rats. J. Pharmacol. Exp. Ther. 287, 860-867 (1998).

47. Pronin, A. N. \& Benovic, J. L. Regulation of the G protein-coupled receptor kinase GRK5 by protein kinase C. J. Biol. Chem. 272, 3806-3812 (1997).

48. Chuang, T. T., LeVine, H. 3rd \& De Blasi, A. Phosphorylation and activation of beta-adrenergic receptor kinase by protein kinase C. J. Biol. Chem. 270, 18660-18665 (1995).

49. Winstel, R., Freund, S., Krasel, C., Hoppe, E. \& Lohse, M. J. Protein kinase cross-talk: membrane targeting of the beta-adrenergic receptor kinase by protein kinase C. Proc. Natl Acad. Sci. USA 93, 2105-2109 (1996).

50. Matthees, E. S. F., Haider, R. S., Hoffmann, C. \& Drube, J. Differential regulation of GPCRs-are GRK expression levels the key? Front. Cell Dev. Biol. 9, 687489 (2021).

51. Cato, M. C. et al. The open question of how GPCRs interact with GPCR kinases (GRKs). Biomolecules 11, 447 (2021).

52. Krasel, C. et al. Dual role of the beta2-adrenergic receptor C terminus for the binding of beta-arrestin and receptor internalization. J. Biol. Chem. 283, 31840-31848 (2008)

53. Gurevich, V. V. et al. Arrestin interactions with G protein-coupled receptors. Direct binding studies of wild type and mutant arrestins with rhodopsin, beta 2 -adrenergic, and $\mathrm{m} 2$ muscarinic cholinergic receptors. J. Biol. Chem. 270, 720-731 (1995).

54. Jones, K. T., Echeverry, M., Mosser, V. A., Gates, A. \& Jackson, D. A. Agonist mediated internalization of $\mathrm{M} 2 \mathrm{mAChR}$ is beta-arrestin-dependent. J. Mol. Signal. 1, 7 (2006)

55. Staus, D. P. et al. Structure of the M2 muscarinic receptor-beta-arrestin complex in a lipid nanodisc. Nature 579, 297-302 (2020).

56. Gomeza, J. et al. Pronounced pharmacologic deficits in M2 muscarinic acetylcholine receptor knockout mice. Proc. Natl Acad. Sci. USA 96, 1692-1697 (1999)

57. Lymperopoulos, A., Rengo, G. \& Koch, W. J. GRK2 inhibition in heart failure: something old, something new. Curr. Pharm. Des. 18, 186-191 (2012). 
58. Mou, L., Gates, A., Mosser, V. A., Tobin, A. \& Jackson, D. A. Transient hypoxia induces sequestration of M1 and M2 muscarinic acetylcholine receptors. J. Neurochem. 96, 510-519 (2006).

59. Torrecilla, I. et al. Phosphorylation and regulation of a $\mathrm{G}$ protein-coupled receptor by protein kinase CK2. J. Cell Biol. 177, 127-137 (2007).

60. Pollok-Kopp, B., Huttenrauch, F., Rethorn, S. \& Oppermann, M. Dynamics of protein kinase $\mathrm{C}$-mediated phosphorylation of the complement $\mathrm{C} 5 \mathrm{a}$ receptor on serine 334. J. Biol. Chem. 282, 4345-4353 (2007).

61. Zhou, X. E. et al. Identification of phosphorylation codes for arrestin recruitment by G protein-coupled receptors. Cell 170, 457-469 e413 (2017).

62. Oakley, R. H., Laporte, S. A., Holt, J. A., Barak, L. S. \& Caron, M. G. Association of beta-arrestin with $\mathrm{G}$ protein-coupled receptors during clathrinmediated endocytosis dictates the profile of receptor resensitization. J. Biol. Chem. 274, 32248-32257 (1999)

63. Baidya, M. et al. Key phosphorylation sites in GPCRs orchestrate the contribution of beta-Arrestin 1 in ERK1/2 activation. EMBO Rep. 21, e49886 (2020).

64. Latorraca, N. R. et al. How GPCR phosphorylation patterns orchestrate arrestin-mediated signaling. Cell 183, 1813-1825 e1818 (2020).

65. Mayer, D. et al. Distinct G protein-coupled receptor phosphorylation motifs modulate arrestin affinity and activation and global conformation. Nat. Commun. 10, 1261 (2019)

66. Komolov, K. E. \& Benovic, J. L. G protein-coupled receptor kinases: past, present and future. Cell Signal. 41, 17-24 (2018).

67. Tesmer, V. M., Kawano, T., Shankaranarayanan, A., Kozasa, T. \& Tesmer, J. J. Snapshot of activated G proteins at the membrane: the Galphaq-GRK2Gbetagamma complex. Science 310, 1686-1690 (2005).

68. Calebiro, D., Koszegi, Z., Lanoiselee, Y., Miljus, T. \& O’Brien, S. G proteincoupled receptor-G protein interactions: a single-molecule perspective. Physiol. Rev. 101, 857-906 (2021).

69. Pandey, S. et al. Intrinsic bias at non-canonical, beta-arrestin-coupled seven transmembrane receptors. Mol. Cell. 81, 4605-4621 (2021).

70. Sanjana, N. E., Shalem, O. \& Zhang, F. Improved vectors and genome-wide libraries for CRISPR screening. Nat. Methods 11, 783-784 (2014).

71. Dicker, F., Quitterer, U., Winstel, R., Honold, K. \& Lohse, M. J. Phosphorylation-independent inhibition of parathyroid hormone receptor signaling by $\mathrm{G}$ protein-coupled receptor kinases. Proc. Natl Acad. Sci. USA 96, 5476-5481 (1999).

72. Miess, E. et al. Multisite phosphorylation is required for sustained interaction with GRKs and arrestins during rapid mu-opioid receptor desensitization. Sci. Signal. 11, eaas9609 (2018).

73. Reichel, M. GPCR kinase knockout cells reveal the impact of individual GRKs on arrestin-binding and GPCR regulation. https://doi.org/10.5281/ zenodo.5764248 (2021).

74. Hothorn, T., Bretz, F. \& Westfall, P. Simultaneous inference in general parametric models. Biometr. J. 50, 346-363 (2008).

75. Hoffmann, C. et al. Fluorescent labeling of tetracysteine-tagged proteins in intact cells. Nat. Protoc. 5, 1666-1677 (2010).

76. Quitterer, U., Pohl, A., Langer, A., Koller, S. \& Abdalla, S. A cleavable signal peptide enhances cell surface delivery and heterodimerization of Ceruleantagged angiotensin II AT1 and bradykinin B2 receptor. Biochem. Biophys. Res. Commun. 409, 544-549 (2011).

77. Rizk, A., Mansouri, M., Ballmer-Hofer, K. \& Berger, P. Subcellular object quantification with Squassh3C and SquasshAnalyst. Biotechniques 59, 309-312 (2015).

78. Rizk, A. et al. Segmentation and quantification of subcellular structures in fluorescence microscopy images using Squassh. Nat. Protoc. 9, 586-596 (2014).

79. Nagel, F. et al. Structural determinants of agonist-selective signaling at the sst(2A) somatostatin receptor. Mol. Endocrinol. 25, 859-866 (2011).

80. Poll, F. et al. Pasireotide and octreotide stimulate distinct patterns of sst2A somatostatin receptor phosphorylation. Mol. Endocrinol. 24, 436-446 (2010).

81. Schroder, R. et al. Applying label-free dynamic mass redistribution technology to frame signaling of $\mathrm{G}$ protein-coupled receptors noninvasively in living cells. Nat. Protoc. 6, 1748-1760 (2011).

82. Malfacini, D. et al. Rational design of a heterotrimeric $\mathrm{G}$ protein alpha subunit with artificial inhibitor sensitivity. J. Biol. Chem. 294, 5747-5758 (2019).

83. Meyrath, M. et al. The atypical chemokine receptor ACKR3/CXCR7 is a broadspectrum scavenger for opioid peptides. Nat. Commun. 11, 3033 (2020).

84. Annala, S. et al. Direct targeting of Galphaq and Galpha11 oncoproteins in cancer cells. Sci. Signal. 12, eaau5948 (2019).
85. Krebs, K. M. et al. Label-free whole cell biosensing for high-throughput discovery of activators and inhibitors targeting $\mathrm{G}$ protein-activated inwardly rectifying potassium channels. ACS Omega 3, 14814-14823 (2018).

86. Wright, S. C. et al. FZD5 is a Galphaq-coupled receptor that exhibits the functional hallmarks of prototypical GPCRs. Sci. Signal. 11, eaar5536 (2018).

\section{Acknowledgements}

We want to thank Ulrike Schiemenz and Nina Kathleen Blum for assistance with the MOPinternalisation studies, Dr. Aurélien Rizk for the help in microscopy data analysis, Prof. Tom Kirchhausen for providing the Rab5-mCherry plasmid, and the Core Facility Flow cytometry of the FLI-Leibniz Institute for Age Research, Jena, for sorting of the stable cell lines.

\section{Author contributions}

J.D. engineered all GRK knockout cells; J.D., R.S.H. and C.H. developed the concept and designed the experiments; J.D., R.S.H., E.S.F.M., M.R., S.B, C.Z., L.K., J.F. and V.W. conducted the experimental work; J.D., R.S.H., E.S.F.M. and M.R. compiled the data; J.Z. designed, conducted and analysed AT1R targeted DMR measurements, supervised by E.K.; S.F. and A.K. planned, conducted and analysed the MOP internalisation experiments with support from E.M.-T.; C.H. supervised the project; S.S. provided phosphosite specific antibodies; J.D., R.S.H., E.S.F.M., M.R. and C.H. wrote the paper; all other authors critically revised the paper and gave final approval.

\section{Funding}

C.H. was supported by the European Regional Development Fund (Grant ID: EFRE HSB 2018 0019), the federal state of Thuringia, the Deutsche Forschungsgemeinschaft (Grants: CRC166, ReceptorLight, project C02 and Polytarget; SFB1278: 316213987, project D02). J.D. is additionally funded by the University Hospital Jena IZKF (Grant ID: MSP10). E.K. gratefully acknowledges the support of this work by the DFG-funded Research Unit FOR2372 with the grants KO 1582/10-1 and KO 1582/10-2. Open Access funding enabled and organized by Projekt DEAL.

\section{Competing interests}

S.S. is the founder and scientific advisor of 7TM Antibodies GmbH, Jena, Germany. The remaining authors declare no competing interests.

\section{Additional information}

Supplementary information The online version contains supplementary material available at https://doi.org/10.1038/s41467-022-28152-8.

Correspondence and requests for materials should be addressed to C. Hoffmann.

Peer review information Nature Communications thanks the anonymous reviewer(s) for their contribution to the peer review of this work. Peer reviewer reports are available.

Reprints and permission information is available at http://www.nature.com/reprints

Publisher's note Springer Nature remains neutral with regard to jurisdictional claims in published maps and institutional affiliations.

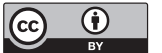

Open Access This article is licensed under a Creative Commons Attribution 4.0 International License, which permits use, sharing, adaptation, distribution and reproduction in any medium or format, as long as you give appropriate credit to the original author(s) and the source, provide a link to the Creative Commons license, and indicate if changes were made. The images or other third party material in this article are included in the article's Creative Commons license, unless indicated otherwise in a credit line to the material. If material is not included in the article's Creative Commons license and your intended use is not permitted by statutory regulation or exceeds the permitted use, you will need to obtain permission directly from the copyright holder. To view a copy of this license, visit http://creativecommons.org/ licenses/by/4.0/.

(C) The Author(s) 2022 